\title{
Research Square \\ Comparison of Complexity of the Switchable Chaotic Systems and its FPGA Implementation
}

Shaohui Yan ( $\nabla$ mortals_ysh@163.com )

Northwest Normal University https://orcid.org/0000-0002-6886-0440

Qiyu Wang

Northwest Normal University

\section{Research Article}

Keywords: Factional-order chaotic system , C0 complexity , SE complexity , Circuit design , FPGA

Posted Date: June 9th, 2021

DOI: https://doi.org/10.21203/rs.3.rs-580646/v1

License: (9) This work is licensed under a Creative Commons Attribution 4.0 International License. Read Full License 


\title{
Comparison of Complexity of the Switchable Chaotic Systems and its FPGA Implementation
}

\author{
Shaohui Yan - Qiyu Wang - Xi Sun · Ertong Wang - Wanlin Shi · \\ Zhenlong Song
}

Received: date / Accepted: date

\begin{abstract}
A four-dimensional chaotic system with complex dynamical properties is constructed via introducing a nonlinear function term. The paper assesses complexity of the system employing equilibrium points, Lyapunov exponent spectrum and bifurcation model. Specially, the coexisting Lyapunov exponent spectrum and the coexisting bifurcation validate the coexistence of attractors. The corresponding complexity characteristics of the system can be analyzed by using $C 0$ and spectral entropy $(S E)$ complexity algorithms, and the most complicated integer-order system is obtained. Furthermore, the circuit which can switch the chaotic attractors is implemented. It is worth noting that the more sophisticated parameters are received by comparing the complexity of the most complicated integer-order chaotic system with corresponding fractional-order chaotic system. Finally, the results of simulation model built in the MATLAB are the same as the hardware verified on the Field-Programmable Gate Array(FPGA) platform, which verify the feasibility of the system.
\end{abstract}

Keywords Factional-order chaotic system $\cdot C 0$ complexity $\cdot S E$ complexity · Circuit design · FPGA

\section{Introduction}

In recent years, chaotic systems with complex dynamical characteristics have been concerned, and they have great potential in engineering applications, such as chaotic

\section{Shaohui Yan ${ }^{凶}$}

College of Physics and Electronic Engineering, Northwest Normal University, Lanzhou, 730070, China

E-mail: mortals_ysh@163.com

Qiyu Wang

College of Physics and Electronic Engineering, Northwest Normal University, Lanzhou, 730070, China synchronization [1-3], image encryption [4-6]and neural networks[7-9]. The analysis of equilibrium point plays a major role in the study of chaos dynamics. Fang et al. [10-12] proposed chaotic systems without equilibrium point. Deng et al. [13] studied chaotic systems that have only one stable equilibrium point but are unstable at infinity. Wang et al. [14] introduced a chaotic system with only one linear equilibrium point that generate the coexistence of one and two wings. Multiple equilibrium points are one of the elements that produce complex dynamical behaviors of the system. In this case, multi-scoll attractors and coexisting attractors can be generated. The phenomenon of multiple vortices and coexisting attractors are a hot topic now. Wang et al. [15] discovered a multi-scroll chaotic system based on step function and sign function and gave its generation mechanism. D. et al. [16] proposed an autonomous system with coexistence of multiple vortices. Liu et al. [17] introduced a new system that could generate multidirection multi-scoll (MDMS) chaotic attractors. Under the certain combination of parameters, different trajectories appear because of various combinations of initial values. While some of the trajectories converge to the identical attractor, some of the trajectories converge to other attractors. These attractors are called coexisting attractors [18]. The system proposed by Ma et al. [19] has 12 attractors of different shapes. The system proposed by Wang et al. [20] has coexistence of asymmetric attractors. Switches with simple design are easy to control in the control system, so they have been widely used. For chaotic systems, switchable chaotic systems have more complicated pseudo-random series than single chaotic systems, and have higher security in chaotic communication and other aspects. A controller is designed on the basis of Spott B system proposed by Lai et al. [21], which can switch trajectory of the sys- 
tem between different chaotic attractors. Zhu et al. [22] proposed the idea based on secure communication and discussed the stability of the switchable system in detail where it was used as the transmitter. To generate the complex chaotic attractors, Yao Sigai [23] designed an analog circuit to switch between the three subsystems. Huang et al. [24] investigated the fractional-order switching system to obtain larger key space. The greater the randomness is, the more difficult it is to restore the sequence. Therefore, in the application of secure communication, the system can be ensured to have better anti-jamming and stronger anti-interception. Ye et al. [25] analyzed the complexity characteristics of Bao chaotic system, Rossler chaotic system and Wien Bridge hyperchaotic system based on $S E$ and $C 0$ algorithms. Ma et al. [26] used chaos graph based on $S E$ complexity to realize the simultaneous change of bifurcation parameters and analyzed the difference of complexity of the system under different initial values. Li et al. [27] showed that $S E$ and $C 0$ complexity algorithms had higher computational accuracy and had more obvious basis for parameter selection of chaos map. Sun et al. [28] proved that the $S E$ algorithm has fewer parameters but higher robustness, and can effectively analyze the complexity of pseudo-random sequences. Chen et al. [29] found that the calculation speed of $C 0$ complexity was faster than that of Lyapunov spectrum, and it consumed less resources. They also concluded that the complexity of fractional-order system was greater than that of the integer-order. Li et al. [30] studied the JafariSpott fractional-order chaotic system with the Adomian decomposition method, and found that the complexity of the fractional-order system was greater than integerorder system after the detection of the complexity with the dispersion entropy. The fractional-order system had an advantageous application prospect. Wang et al. [31] studied the fractional-order multicavity chaotic map and showed that the fractional-order system has richer dynamical characteristics, higher complexity and more uniform distribution. However, in this paper, the complexity of most complex fractional-order subsystem is studied based on the $C 0$ and $S E$ complexity algorithms. Both fractional-order and integer- order chaotic circuits can be switchable, which may provide a better fractional-order model for chaotic applications.

The structure of article is organized as follows. In Sect. 2 , a four-dimensional chaotic system is proposed, then numerical simulations and dynamical analysis are illustrated. Moreover, we compare the complexity of the different subsystems. A switchable integer-order circuit is implemented in Sect. 3. In Sect. 4, the complexity of fractional-order system is analyzed and the analog circuit is completed. In Sect. 5, the hardware circuit is realized based on FPGA. Finally, all the results are summarized.

\section{Chaotic system and dynamical analysis}

The phase-orbit diagram is a record of the system trajectory and reflects the change of the system state. The dynamic behaviors of the system are judged by observing the projection of phase diagram on the plane. This is the most direct way to observe the dynamic behaviors of a system [32]. For better analyzing its chaotic characteristics, we proceed a research about equilibrium points, Lyapunov exponent spectrum, bifurcation model and complexity. The coexistence of bifurcation diagrams and the coexistence of Lyapunov exponents are used to illustrate coexisting attractors. The Lyapunov exponent spectrum is a useful tool for measuring whether the system is chaotic or hyperchaotic. In particular, systems with one positive Lyapunov exponent are chaotic, and those with two or more positive Lyapunov exponents are hyperchaotic [32]. The coexistence model of bifurcation is substantially the mapping of the coexistence attractors on the bifurcation plane [33].

\subsection{System model}

Based on the proposed system [34], a novel 4D system which is the addition of to the equation of the original system is conducted in Eq. (1):

$\left\{\begin{array}{l}\dot{x}=-a x+y z-b z+g(x, y) \\ \dot{y}=2 y-x z \\ \dot{z}=x y+w-c z \\ \dot{w}=-3 z-w\end{array}\right.$

where three parameters $a=1, b=0, c=3, x, y$ and $z$ are state variables. Meanwhile, the initial value is $x_{0}=$ $(2,4,1,3)$. To system $(1), g(x, y)$ can be substituted for $z^{2}$ or $w^{2}$ or $-x y$ or $y w$. Fixed $a, b$ and $c$ unchanged, the chaotic attractors of the system appear as double-scoll when $g(x, y)$ is $z^{2}$, while $g(x, y)$ is $w^{2}$, the attractors exhibit single vortex. When $g(x, y)$ is equal to $-x y$, double-scoll attractors are performed. When $g(x, y)$ is $y w$, the chaotic system shows four-scoll attractors. For the convenience of description, different systems are abbreviated as system (2), system (3), system (4) and system (5), respectively.

\subsection{Equilibrium points and stability analysis}

The number and stability of equilibrium points play an important role in the dynamic behavior of the system. 
The existence of multiple equilibrium points is also a vital factor for the generation of multi-scoll attractors and coexisting attractors. The equilibrium points of system (2) can be calculated by allowing $\dot{x}=\dot{y}=\dot{z}=0$. Under a set of fixed system parameters $a=1, b=0, c$ $=3$, the equilibrium points of system $(2)$ are $P_{0}(0,0$, 0, 0), $P_{1}(3.4641,-1.9503,-1.1260,3.3781), P_{2}(-3.4641$, $3.7678,-2.1753,6.5260), P_{3}(3.4641,1.9503,1.1260$, $3.3781)$ and $P_{4}(-3.4641,-3.7678,2.1753,-6.5260)$. The equilibrium points of system $(3)$ are $E_{0}(0,0,0,0)$, $E_{1}(3.4641,-0.984,0.5681,1.7044), E_{2}(3.4641,-0.984$, $0.5681,-1.7044)$. The equilibrium points of system (4) are $S_{0}(0,0,0,0), S_{1}(-3.4641,-0.8730,0.504,-1.5121)$, $S_{2}(3.4641,6.8730,3.9681,-11.9044), S_{3}(3.4641,-0.8730$, $-0.504,1.5121), S_{4}(-3.4641,6.8730,-3.9681,11.9044)$. For system (5), the only one equilibrium point is $M_{0}(0$, $0,0,0)$. The eigenvalues corresponding to $M_{0}$ are $\lambda_{1,2}=$ $-2 \pm 1.4142 i, \lambda_{3}=-1$ and $\lambda_{4}=2$. Therefore, $M_{0}$ is an unstable saddle focus.

By linearizing the equilibrium point of system (1), the Jacobian matrix is Eq. (2):

$J=\left[\begin{array}{cccc}1 & z & y+2 z & 0 \\ -z & 2 & -x & 0 \\ y & x & -3 & 1 \\ 0 & 0 & -3 & -1\end{array}\right]$

The initial value is $(2,4,1,3)$. As can be seen from Table 1, the eigenvalues and stability of system (1) are shown. Similarly, obtaining the proper values and stability of system (3) and (4), we also present in Table 1.

\subsection{Dynamic characteristic analysis of subsystems}

The phase diagrams of system (2) are shown in Fig.1, which presents as a double-scroll. Keeping other parameters uniform as mentioned above, the parameter $c$ is altered in the range of 3 to 7 , the corresponding Lyapunov exponent spectrum and bifurcation diagram of system (2) are plotted in Fig. 2(a) and (b). According to the figure, the state of system (2) switches between chaos and period with the change of parameter $c$. While the parameter $c \in[3,5.65) \cup(5.95,6.30)$, one of the Lyapunov exponents of system (2) is greater than 0 , under two regions the system condition is chaotic. When $c$ for the range $[5.65,5.95]$ and $[6.30,7]$, the maximum Lyapunov exponent spectrum of system (2) is approximately 0 , showing a periodic state. The Fig. 2(c) obtains the coexisting Lyapunov exponent spectrum of state variable $x$ changing with $c$. It is in accordance with the coexisting bifurcation diagram (Fig. 2(d)) corresponding to the initial value $x_{0}=(2,4,1,3)$ and $x_{1}=(1,2,3,4)$, respectively. When $c=5.90$ and 6.50 , the system (2) expresses the coexistence of periodic and chaotic attractors as shown in Fig. 2(e) and the coexistence of two periodic attractors as shown in Fig. 2(f).

In the same system, $S E$ and $C 0$ algorithms are used to calculate the complexity characteristics of $x$ sequence, and the complexity in chaotic state is greater than that in periodic state [35]. $C 0$ complexity and $S E$ complexity (Fig. 3(a) and (b)) corresponding to Lyapunov exponent spectrum and bifurcation diagram, it can be found that when parameter $c$ is in a periodic state, the complexity decreases significantly, while parameter $c$ is in a chaotic state, the complexity has obvious increase. Taking $c=4.10$ as an example, its corresponding phase diagram which represents chaotic attractor is shown in Fig. 3(c). However, as shown in Fig. $3(\mathrm{~d}), c=5.90$ exhibits a periodic attractor. Finally, all results are consistent.

The Fig. 4 shows its attractor portraits under the parameter conditions $a=1, b=0, c=3$. The Lyapunov exponent spectrum and bifurcation diagram of system (3) are obtained by increasing the control parameter $c$ from 3 to 7, which are shown in Fig. 5(a) and (b). As shown in Fig. 5(c) and (d), the coexistence of Lyapunov exponent spectrum and bifurcation diagram of system (3) vary with parameter $c$, and select $(2,4,1,3)$ and $(1$, $2,3,4)$ as initial values, respectively. When $c$ is 4.40 , two chaotic attractors coexist (Fig. 5(e)), and two periodic attractors exist (Fig. $5(\mathrm{f}))$ under $c=4.23$. What's more, coexisting attractors phases exhibit the state of chaotic system with the different parameter $c$. The dynamical behaviors of chaotic system with coexisting attractors are more complex, which is more suitable for image encryption, information security and other applications. Then, corresponding to complexity diagram $C 0$ and $S E$ complexity diagram (Fig. 6(a) and (b)), when the regions of $\mathrm{c}$ are chosen to $[4,4.30)$ and $(4.70$, $7]$, the Lyapunov exponents are less than 0 . The system behaves as a periodic state, accordingly, the reduction of complexity is apparent. Taking $c=4.20$, the corresponding phase diagram is shown in Fig. 6(c). Within $c \in[3,4] \cup[4.30,4.70]$ one of the Lyapunov exponents is greater than 0 , the system is in a chaotic state and the complexity value is extremely high, which is basically in line with the simulation results of the corresponding Lyapunov exponent spectrum and bifurcation diagram. The corresponding phase diagram with parameter $c=$ 3.30 is shown in Fig. 6(d). The attractor is periodic.

From Fig. 7(a), (b) and (c), it is observed the chaotic attractors of system (4). When parameter $c$ is increased or decreased in the range of 3 to 7 , the Lyapunov exponent spectrum and bifurcation diagram are plotted in Fig. 8(a) and (b). The system enters the pe- 
Table 1 Equilibrium points and corresponding eigenvalue and its stability

\begin{tabular}{llll}
\hline Systems & Equilibrium points & Eigenvalues & Stability \\
\hline \multirow{4}{*}{$(2)$} & $P_{0}$ & $\lambda_{1,2}=-2 \pm 1.4142 i, \lambda_{3}=-1, \lambda_{4}=2$ & unstable saddle focus \\
& $P_{1}$ and $P_{2}$ & $\lambda_{1,2}=1.0255 \pm 3.1718 i, \lambda_{3}=-3.9602, \lambda_{4}=-1.0907$ & unstable saddle focus \\
& $P_{3}$ and $P_{4}$ & $\lambda_{1,2}=0.0602 \pm 4.0457 i, \lambda_{1,2}=-1.5602 \pm 0.7055 i$ & unstable \\
$(3)$ & $E_{0}$ & $\lambda_{1,2}=-2 \pm 1.4142 i, \lambda_{3}=-1, \lambda_{4}=2$ & unstable saddle focus \\
& $E_{1}$ & $\lambda_{1,2}=0.3616 \pm 3.1868 i, \lambda_{1}, 2=-1.3696 \pm 1.0831 i$ & unstable \\
& $E_{2}$ & $\lambda_{1,2}=-1.4320 \pm 2.7786 i, \lambda_{3}=-1.9521, \lambda_{4}=-0.8320$ & stable node focus \\
& $S_{0}$ & $\lambda_{1,2}=-2 \pm 1.4142 i, \lambda_{3}=-1, \lambda_{4}=2$ & unstable saddle focus \\
& $S_{1}$ & $\lambda_{1,2}=-0.4508 \pm 3.4015 i, \lambda_{3}=-2.8587, \lambda_{4}=0.7602$ & unstable saddle focus \\
& $S_{2}$ & $\lambda_{1,2}=-6.2973 \pm 5.1048 i, \lambda_{1,2}=4.7973 \pm 3.5522 i$ & unstable \\
& $S_{3}$ & $\lambda_{1,2}=-1.8276 \pm 2.6141 i, \lambda_{1,2}=0.3276 \pm 1.8989 i$ & unstable \\
& $S_{4}$ & $\lambda_{1,2}=3.2938 \pm 7.5099 i, \lambda_{3}=-11.6174, \lambda_{4}=2.0298$ & unstable \\
\hline
\end{tabular}

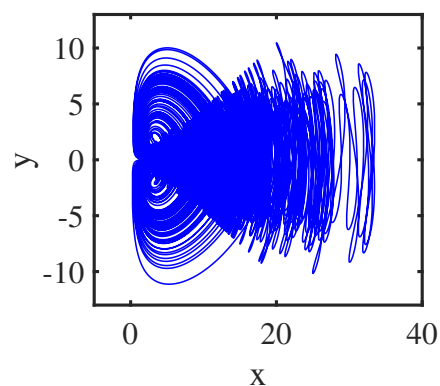

(a)

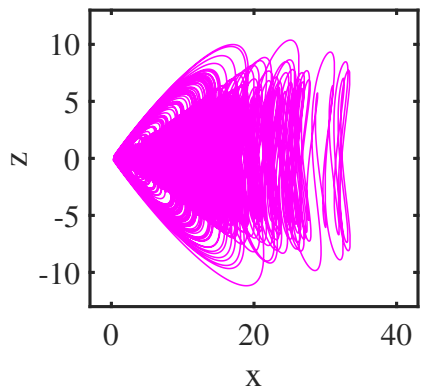

(b)

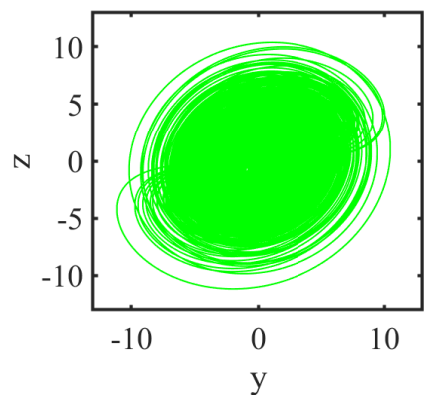

(c)

Fig. 1 Phase diagrams of system (2) with $a=1, b=0$ and $c=3$. a $x-y$ plane; $\mathbf{b} x-z$ plane; c $y-z$ plane

riodic state from chaos through the way of inverse period doubling bifurcation. To illustrate the intricate dynamical behavior, the plots in Fig. 8(c) and (d) present regions where different attractors coexist. When $c$ is chosen to 6.90 , system (4) is characterized by the coexistence of two periodic attractors (see Fig. 8(e) for more details), meanwhile, when $c$ is chosen to 6.35 , the coexistence of periodic and chaotic attractors is exhibited in Fig. 8(f). $C 0$ complexity and $S E$ complexity (Fig. $9(\mathrm{a})$ and (b)) corresponding to the figure can be found that when the parameter $c$ belongs to $[3,6.20]$, two Lyapunov exponents are greater than zero. The system is in a state of hyperchaos, accordingly, complexity value is turned up. For example, $c=5.50$, its corresponding phase diagram is shown in Fig. 9(c). Choosing the interval to encrypt the parameters can be better applied to secure communication. On the contrary, when the range of $c$ is $(6.20,7]$, the maximum Lyapunov exponent is approximately 0 , and the system is in a periodic state. In this case, the corresponding complexity values are lower. Take $c=6.50$ as an example, and the corresponding phase portrait is shown in Fig. 9(d). From the above-mentioned analysis, it can be obtained that the complexity of the same system in the hyperchaotic state is greater than that in the periodic state.

The system (5) is solved with the attractor diagrams plotted in Fig. 10. When parameter $c$ changes from 3 to 7 , the Lyapunov exponent spectrum and bifurcation diagram of system (5) are described by Fig. 11(a) and (b). Lines of different colors represent different $L E$ values. Then, Fig. 11(c) and (d) explain that the coexisting attractors appear in system (5) under the same set of initial values. When $c$ is 5.30 , it is represented by the coexistence of two chaotic attractors, and $c=6.25$ can be found the coexistence of periodic and quasi-periodic attractors. The $x-y-z$ diagrams of coexisting attractors are seen in Fig. 11(e) and (f). Take a closer look at $C 0$ complexity and $S E$ complexity (Fig. $12(\mathrm{a})$ and $(\mathrm{b}))$, which can see that when the parameter $c$ for $[3,6.20)$, the complexity value becomes larger. For example, $c$ is the equal of 5.40, its corresponding phase diagram is shown in Fig. 12(c), which is characterized by chaotic attractor. When the parameter $c$ belongs to $[6.20,7]$, complexity decreased obviously, such as $c=$ 6.45 , its corresponding phase diagram appears as periodic attractor, as shown in Fig. 12(d). The simulation 


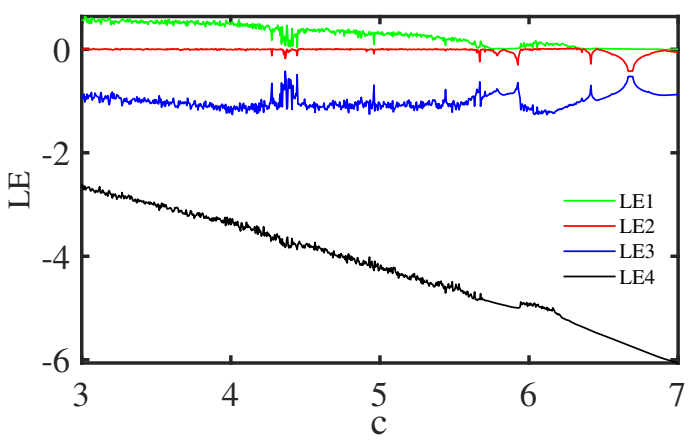

(a)

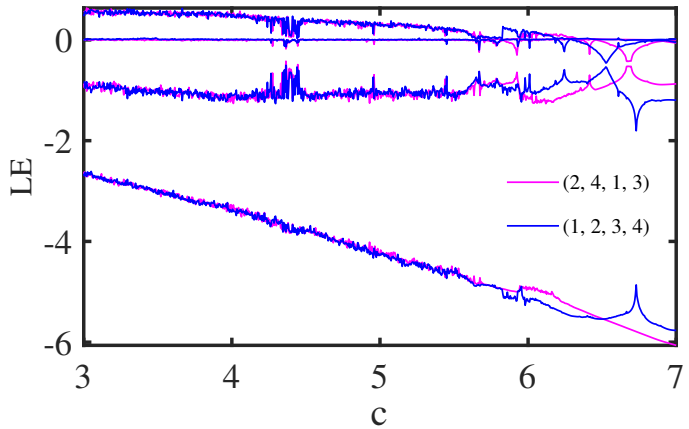

(c)

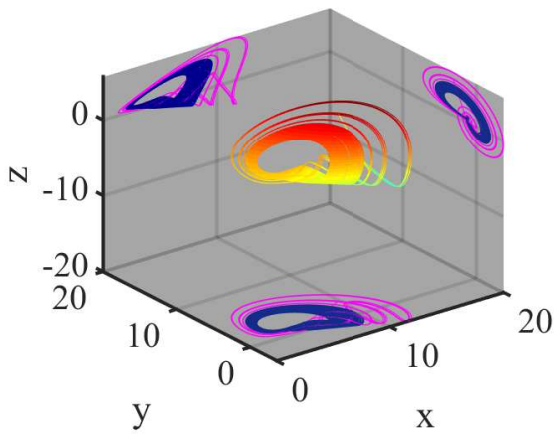

(e)

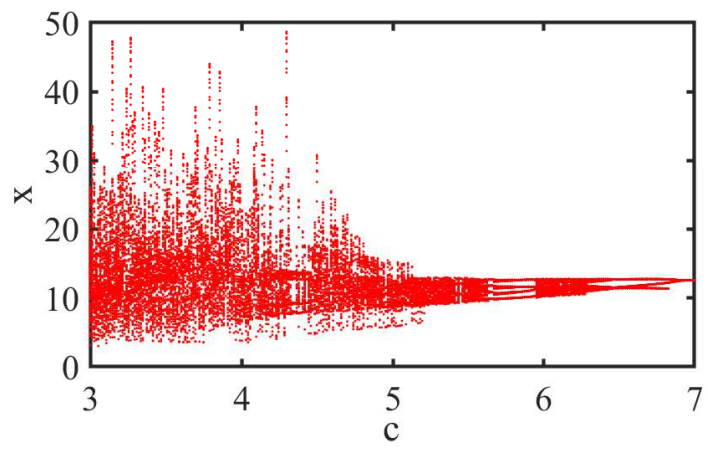

(b)

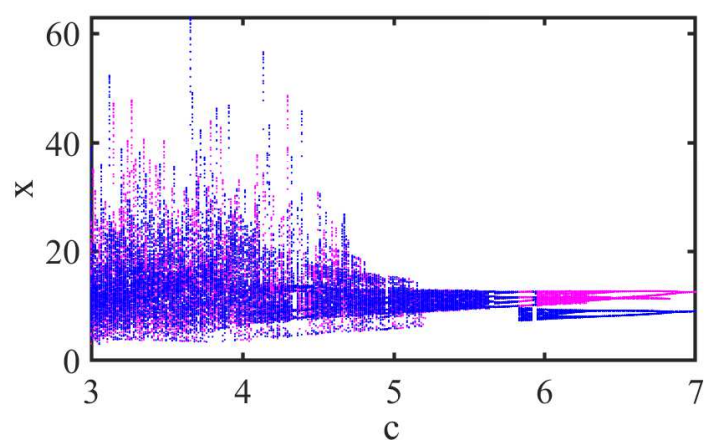

(d)

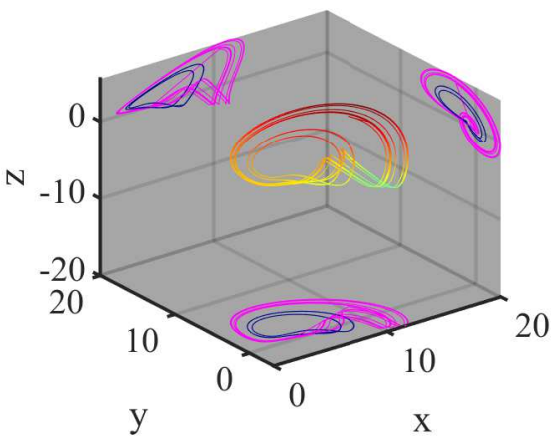

(f)

Fig. 2 Dynamical behaviors varying with parameter [3, 7]. a Lyapunov exponents; b bifurcation diagram; c coexisting Lyapunov exponent spectrum of $x_{0}=(2,4,1,3)$ and $x_{1}=(1,2,3,4)$; d coexisting bifurcation diagram of $x_{0}=(2,4,1,3)$ and $x_{1}=(1,2$, $3,4)$; e the coexistence of periodic and chaotic attractors $(c=5.90) ; \mathbf{f}$ the coexistence of two periodic attractors $(c=6.50)$

results of Lyapunov exponent spectrum and bifurcation diagram are basically in the same way.

\subsection{Comparative analysis of complexity of different systems}

The measurement of system complexity is a significant means to study the dynamic characteristics of chaotic systems, which reflects the security of the system to a certain extent. The closer the chaotic sequence is to the random sequence, the higher the complexity value is and the higher the corresponding security will be [36]. For the different systems studied in this paper, the maximum $C 0$ and $S E$ complexity are compared and analyzed in Table 2. According to Table 2, the data analysis on the maximum $C 0$ and $S E$ complexity show that system (4) is the most complicated, which is basically in 


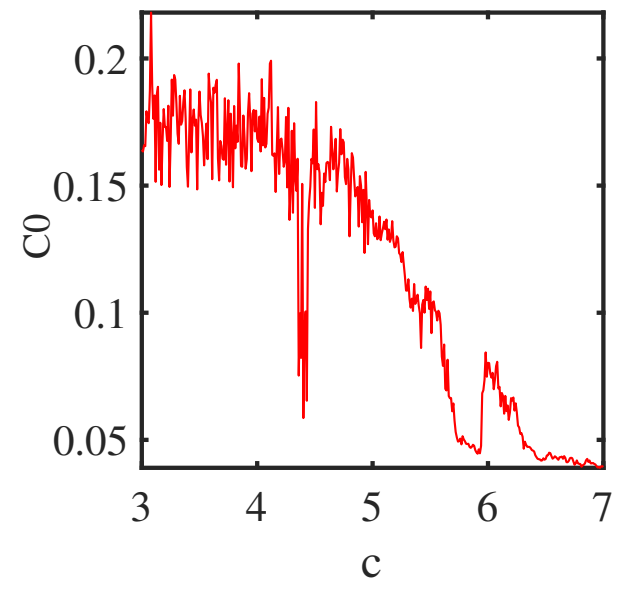

(a)

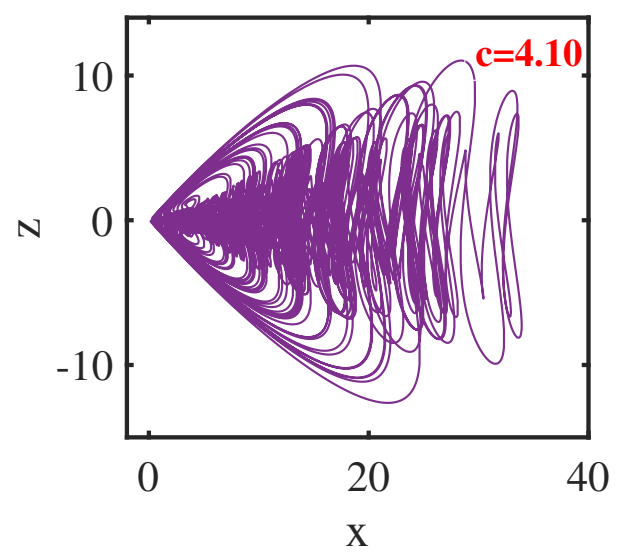

(c)

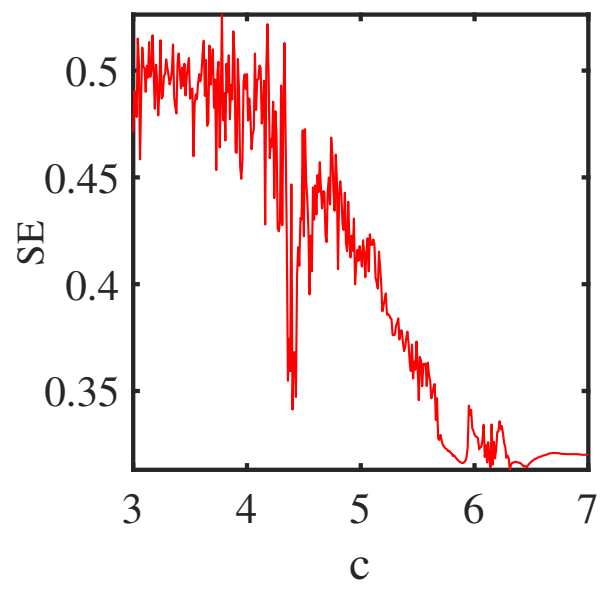

(b)

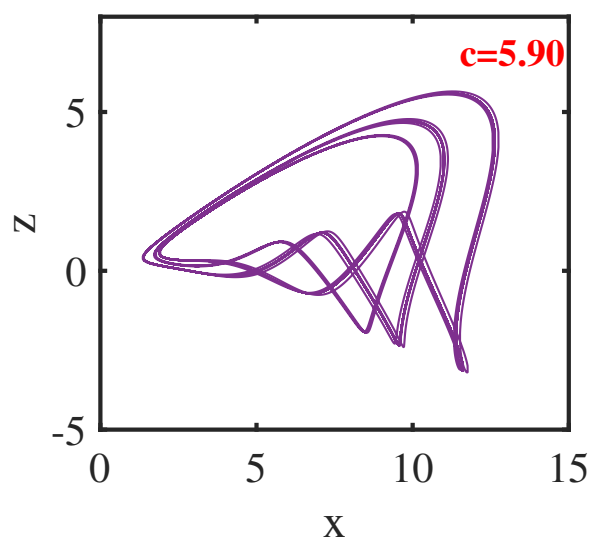

(d)

Fig. 3 The complexity and corresponding phase diagrams of system $(2)$ with $x_{0}=(2,4,1,3)$. a $C 0$ complexity; b $S E$ complexity; c $c=4.10 ; \mathbf{d} c=5.90$

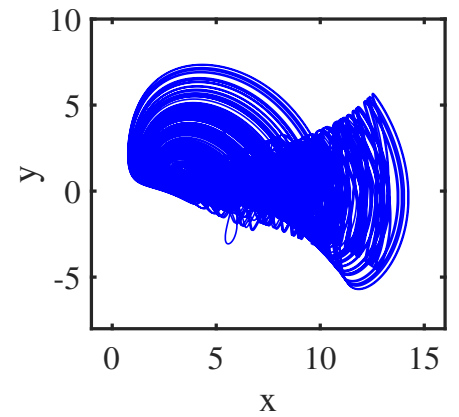

(a)

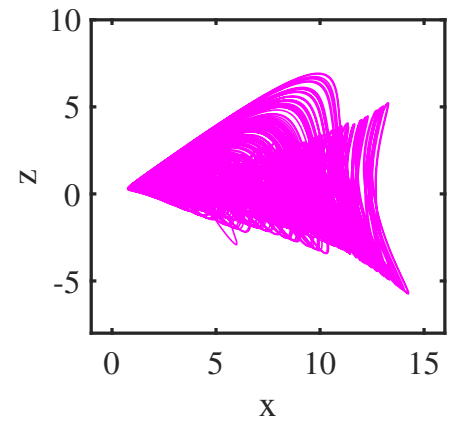

(b)

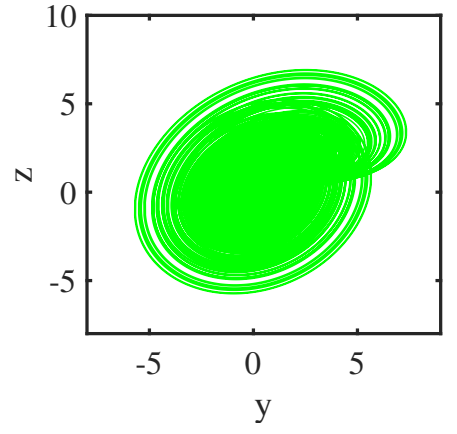

(c)

Fig. 4 Attractor portraits of system (3) with $a=1, b=0$ and $c=3$. a $x-y$ plane; $\mathbf{b} x-z$ plane; $\mathbf{c} y-z$ plane. 


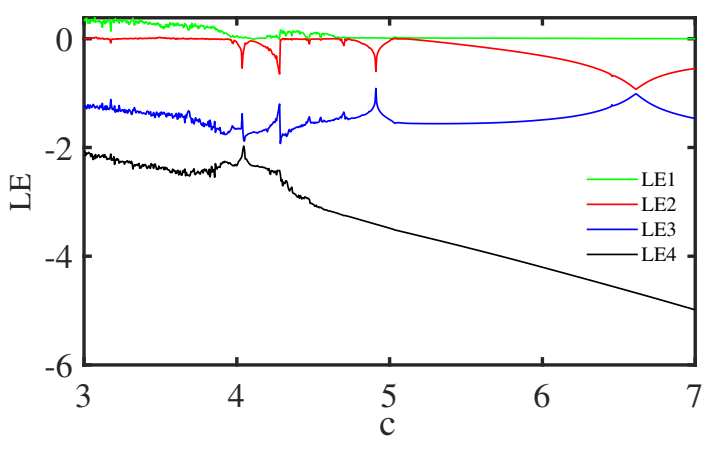

(a)

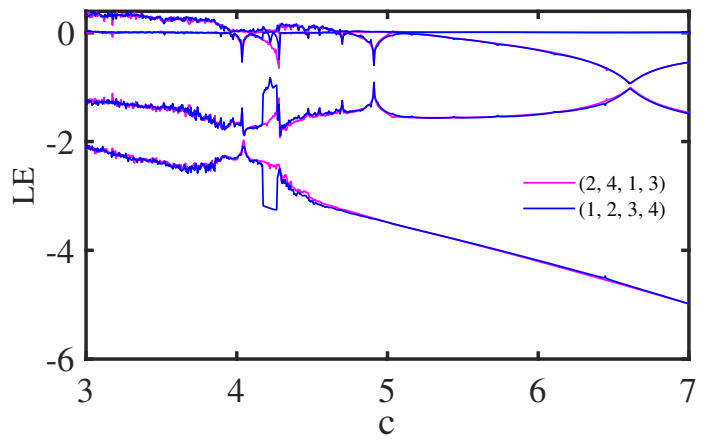

(c)

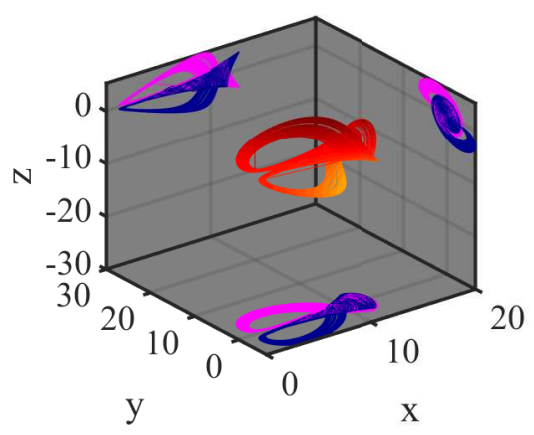

(e)

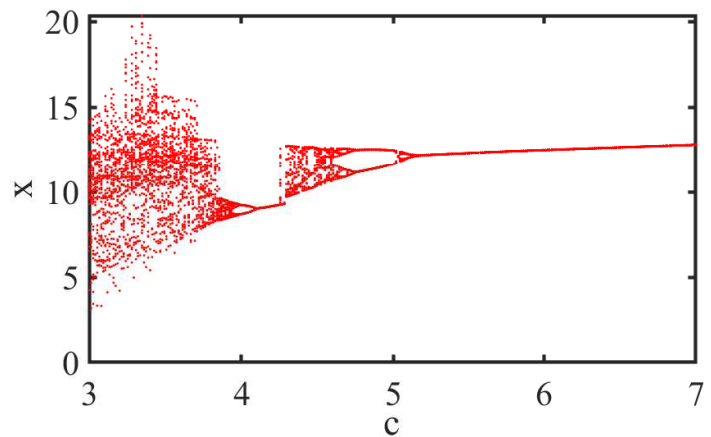

(b)

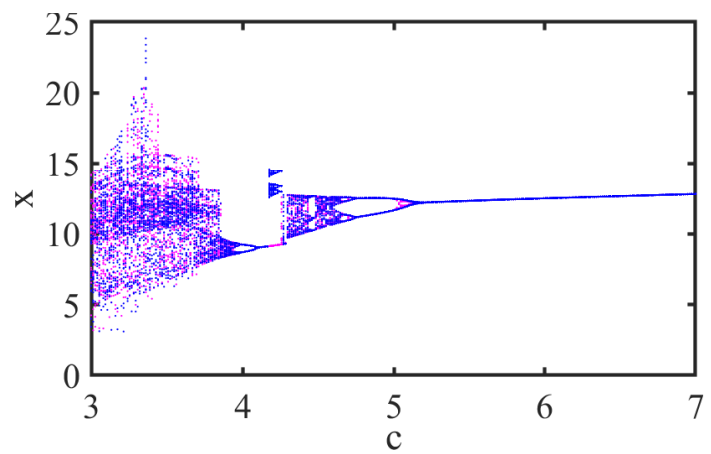

$(\mathrm{d})$

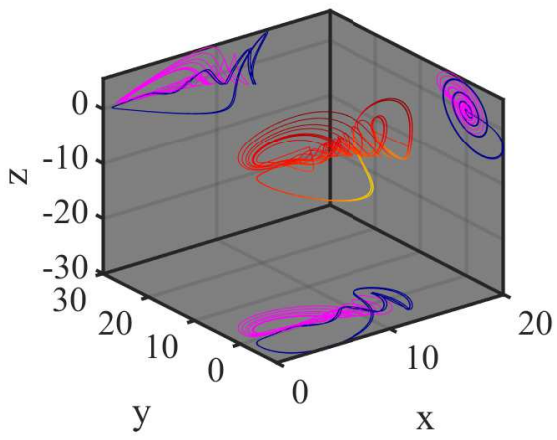

(f)

Fig. 5 Dynamical behaviors varying with parameter from 3 to 7 and initial values as $x_{0}=(2,4,1,3)$ and $x_{1}=(1,2,3,4)$. a Lyapunov exponents; $\mathbf{b}$ bifurcation diagram; c coexisting Lyapunov exponent spectrum; $\mathbf{d}$ coexisting bifurcation diagram; e the coexistence of two chaotic attractors $(c=4.40) ; \mathbf{f}$ the coexistence of two periodic attractors $(c=4.23)$

Table 2 Comparison of complexity of different systems

\begin{tabular}{llll}
\hline System & $g(x, y)$ & $C 0_{\max }$ & $S E_{\max }$ \\
\hline$(2)$ & $z^{2}$ & 0.21 & 0.53 \\
$(3)$ & $w_{2}$ & 0.08 & 0.34 \\
$(4)$ & $-x y$ & 0.36 & 0.65 \\
$(5)$ & $y w$ & 0.27 & 0.61 \\
\hline
\end{tabular}

accordance with the analysis results of various characteristics of the system.

\section{Circuit design and simulation of the system}

The circuit schematic diagram of system (1) as shown in Fig. 13, the circuit design and simulation are accomplished via the Multisim simulation software. Specifically, the switching from system (2) to system (5) can be implemented by the switches $S 1$ to $S 6$ in the ana$\log$ circuit. Four-way analog operation circuit models achieving state variables $x, y, z$ and $w$, analog multiplier 3554AM, and operational amplifier allowable voltage of $\pm 15 \mathrm{~V}$ are set. According to the schematic diagram, the 


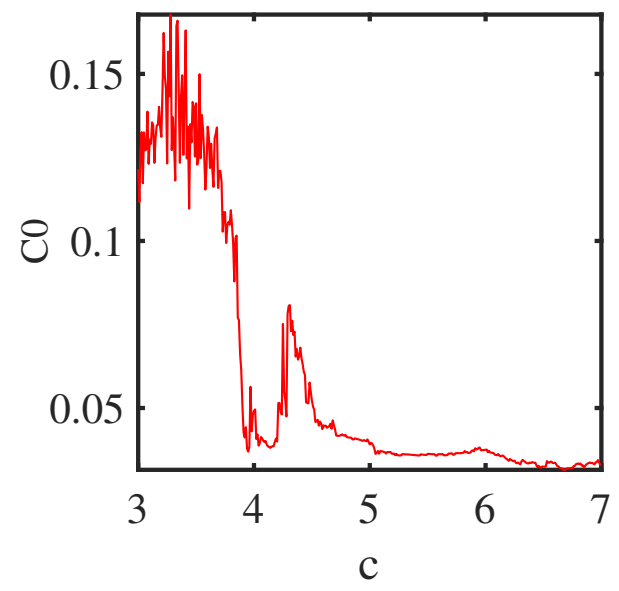

(a)

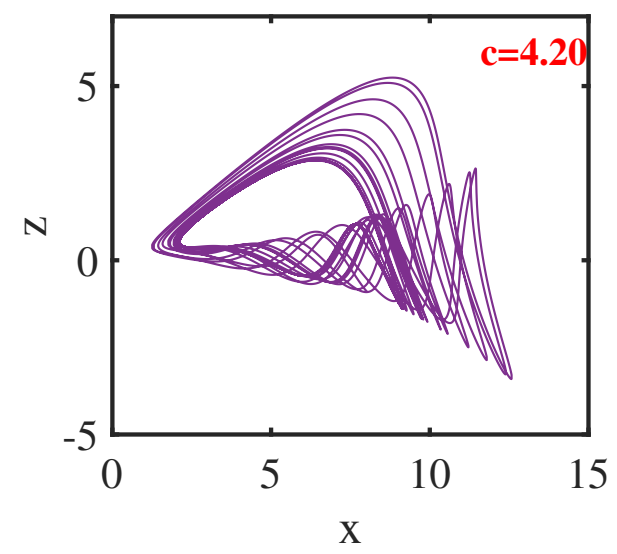

(c)

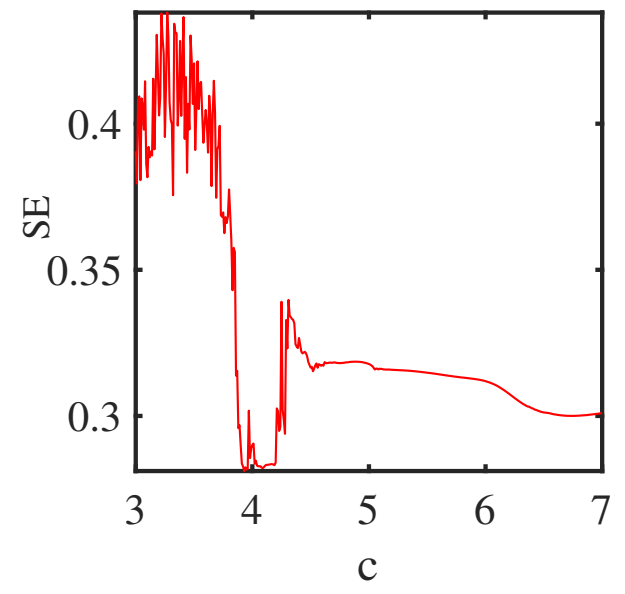

(b)

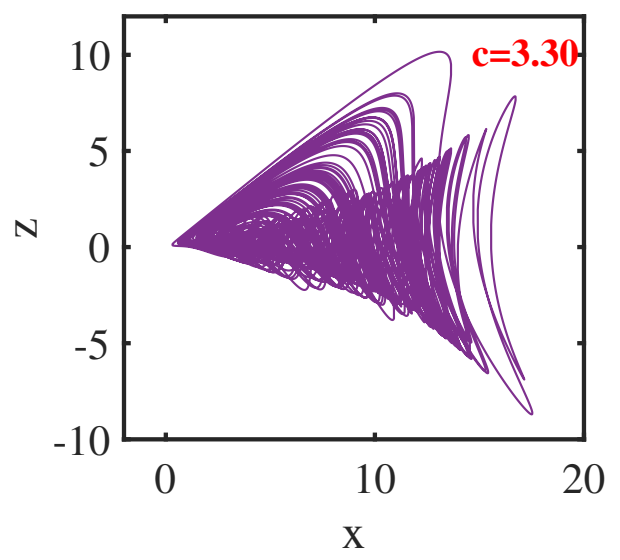

(d)

Fig. 6 The complexity and corresponding attractors portraits of system (3) with $x_{0}=(2,4,1,3)$. a $C 0$ complexity; b $S E$ complexity; $\mathbf{c} c=4.20 ; \mathbf{d} c=3.30$

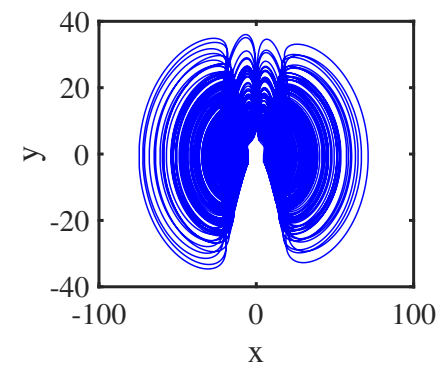

(a)

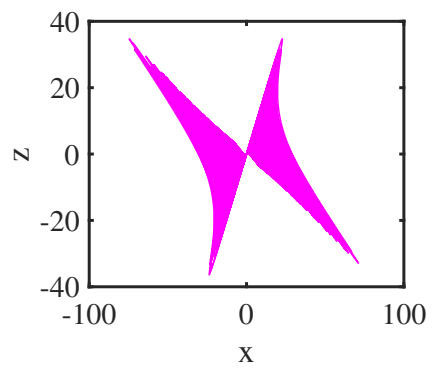

(b)

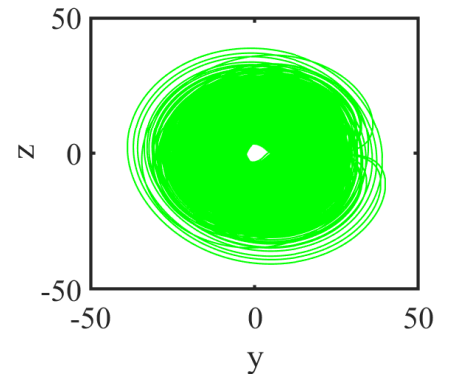

(c)

Fig. 7 Attractor diagrams of system (4) with $a=1, b=0$ and $c=3$. a $x-y$ plane; b $x-z$ plane; c $y-z$ plane 


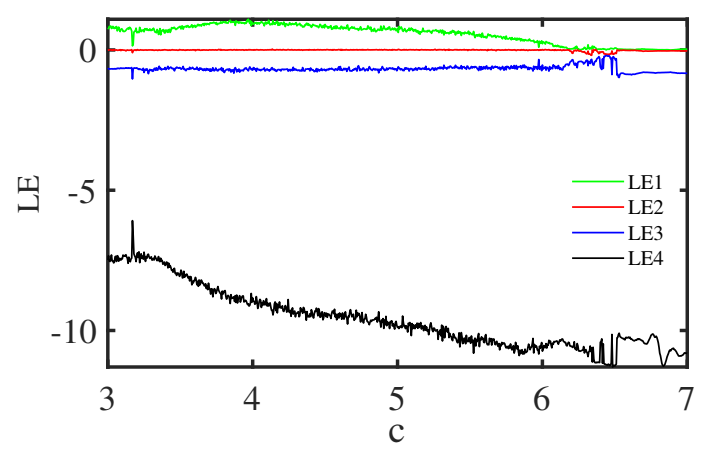

(a)

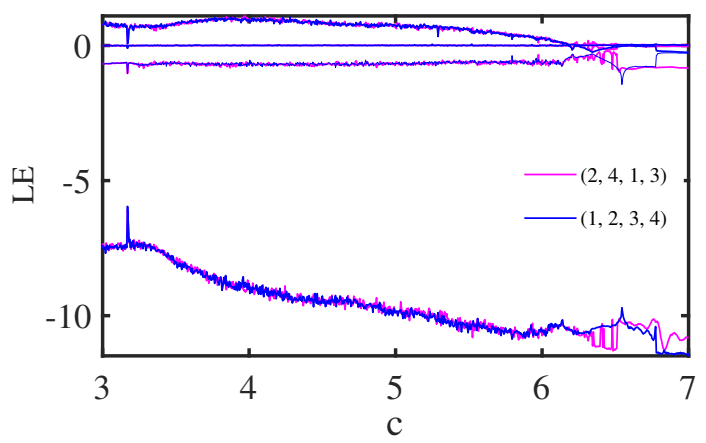

(c)

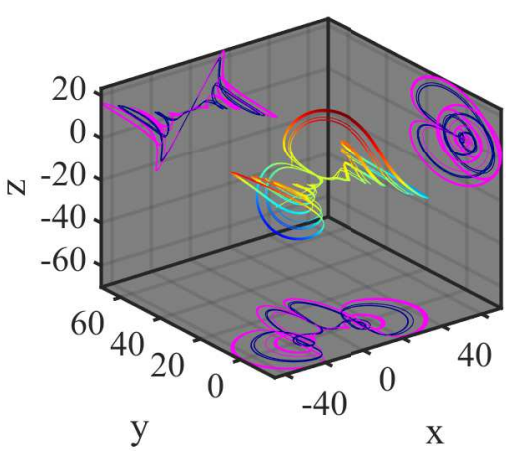

(e)

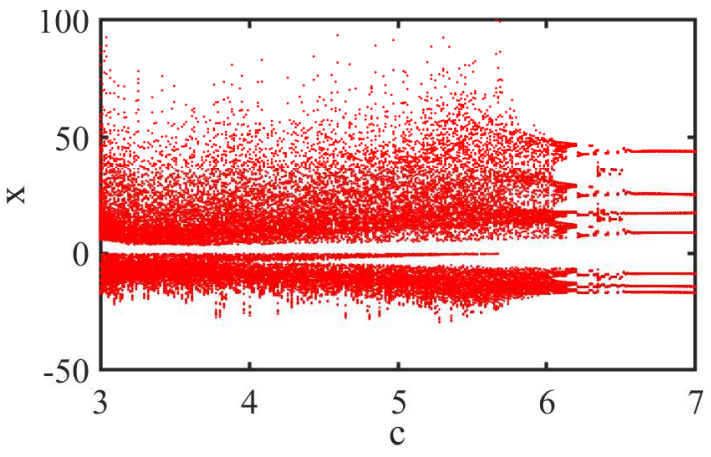

(b)

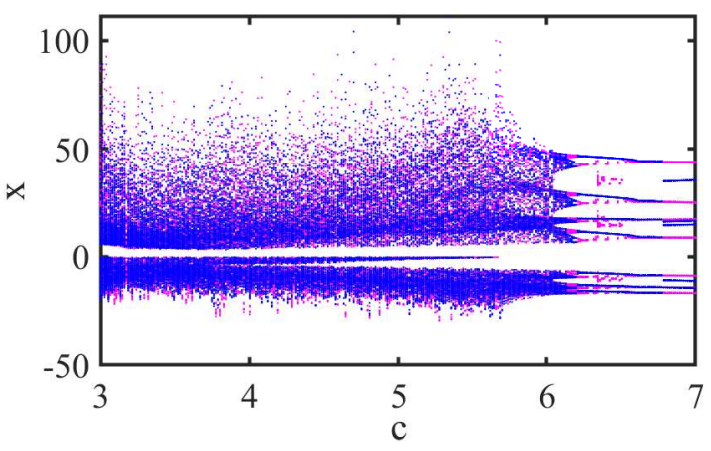

(d)

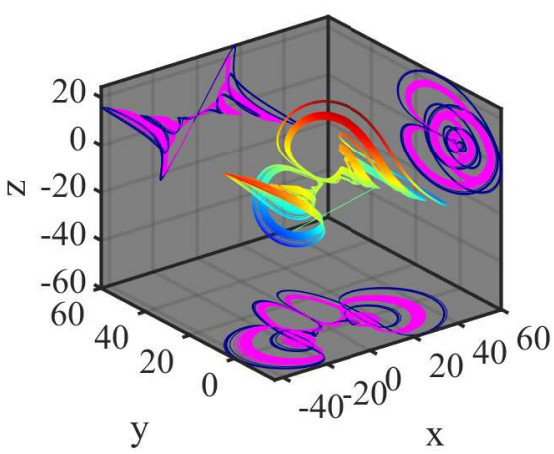

(f)

Fig. 8 Lyapunov exponent spectrum showing in $\mathbf{a}$; bifurcation diagram in $\mathbf{b}$; coexisting Lyapunov exponent spectrum in $\mathbf{c}$ and coexisting bifurcation diagram in $\mathbf{d}$ with $x_{0}=(2,4,1,3)$ and $x_{1}=(1,2,3,4)$; e the coexistence of two periodic attractors $(c=$ $6.90) ; \mathbf{f}$ the coexistence of periodic and chaotic attractors $(c=6.35)$

corresponding state equation is (3)

$$
\left\{\begin{array}{l}
\dot{x}=-\frac{1}{R_{1} C_{1}} x+\frac{1}{R_{2} C_{2}} y z-\frac{1}{R_{3} C_{3}} z+\frac{1}{R_{4} C_{4}} g(x, y) \\
\dot{y}=\frac{2}{R_{5} C_{5}} y-\frac{1}{R_{6} C_{6}} x z \\
\dot{z}=\frac{1}{R_{7} C_{7}} x y+\frac{1}{R_{8} C_{8}} w-\frac{1}{R_{9} C_{9}} z \\
\dot{w}=-\frac{3}{R_{10} C_{10}} z-\frac{1}{R_{11} C_{11}} w
\end{array}\right.
$$

As shown in Table 3, the switches that need to be turned off in the circuit and their corresponding circuit parameters are distinct. The results obtained through Multisim simulation are shown in Fig. 14. By comparing the results in Fig. 1(a), Fig. 4(a), Fig. 7(a), Fig. 10(a) and Fig. 14, it can be found that the phase portraits observed from analog circuit and numerical study are all in accordance, which verify the feasibility of the system. 


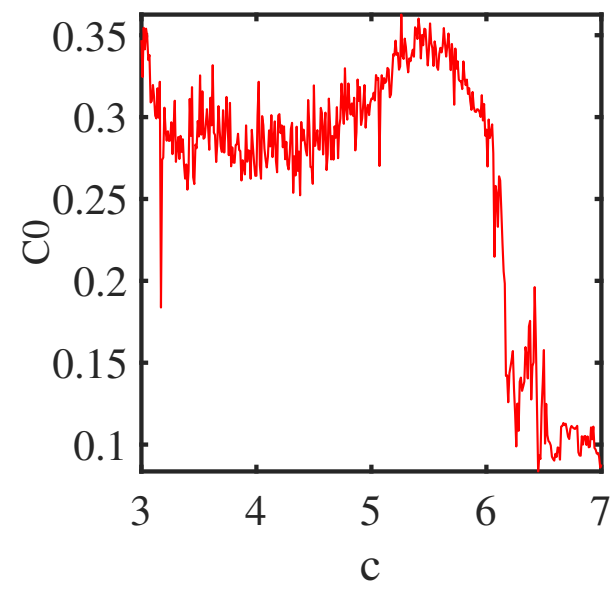

(a)

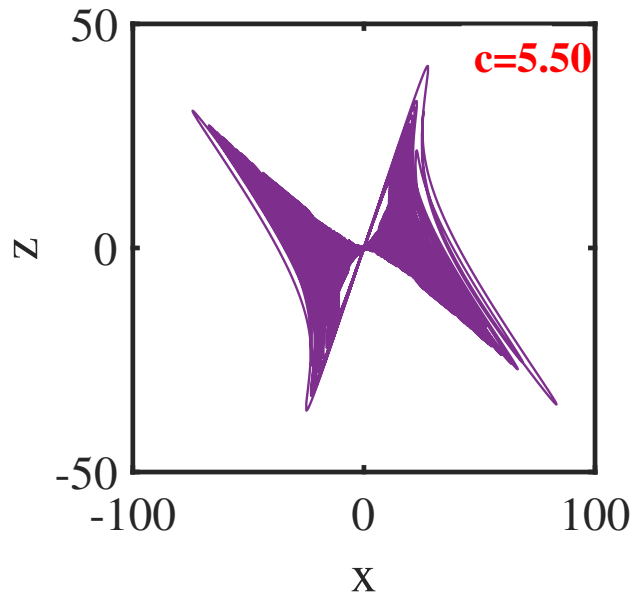

(c)

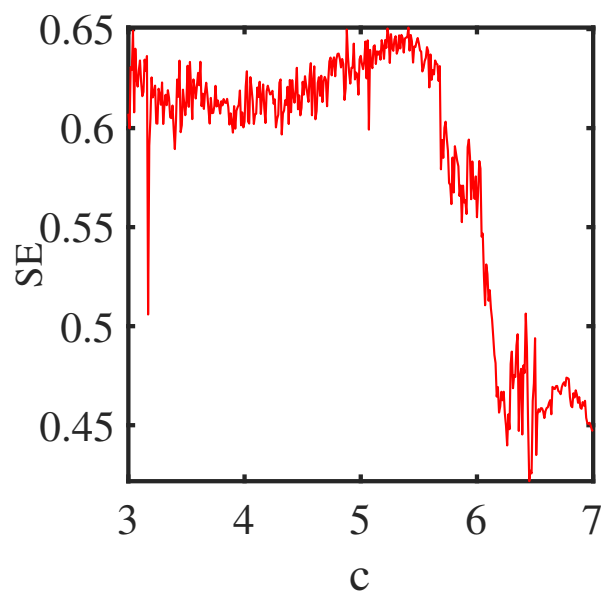

(b)

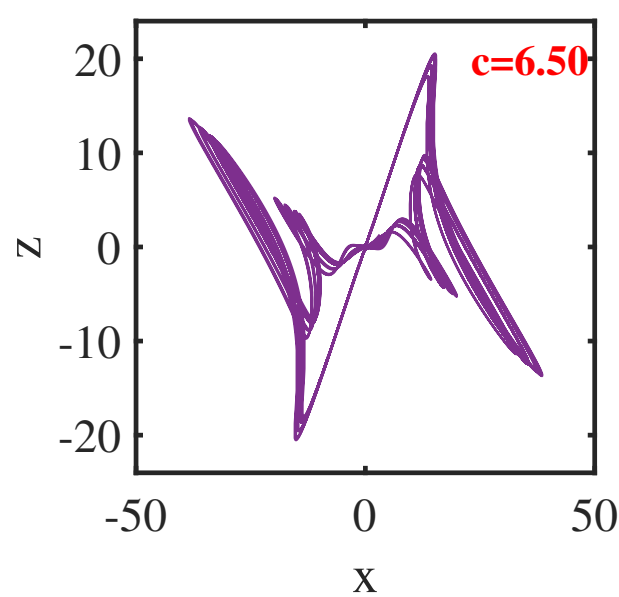

(d)

Fig. 9 The complexity and corresponding attractors portraits of system $(4) x_{0}=(2,4,1,3)$. a $C 0$ complexity; b $S E$ complexity; c $c=5.50 ; \mathbf{d} c=6.50$

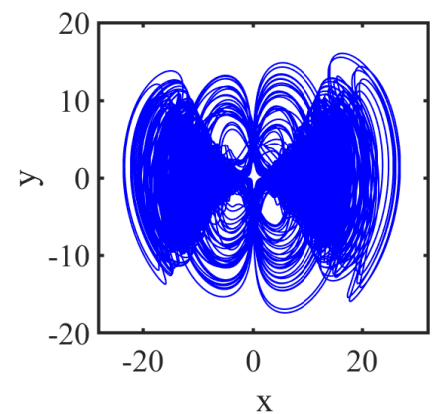

(a)

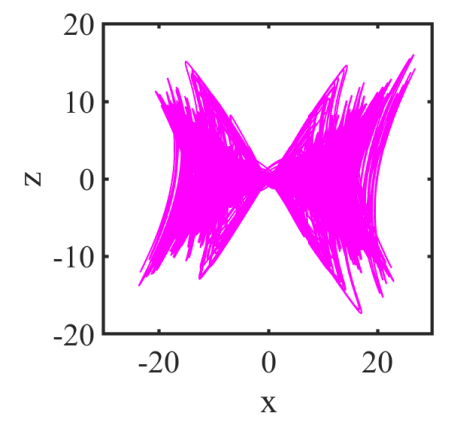

(b)

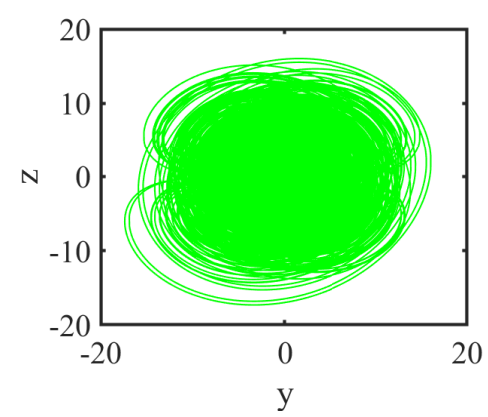

(c)

Fig. 10 Chaotic attractors of system (5) with $a=1, b=0$ and $c=3$. a $x-y$ plane; $\mathbf{b} x-z$ plane; $\mathbf{c} y-z$ plane 


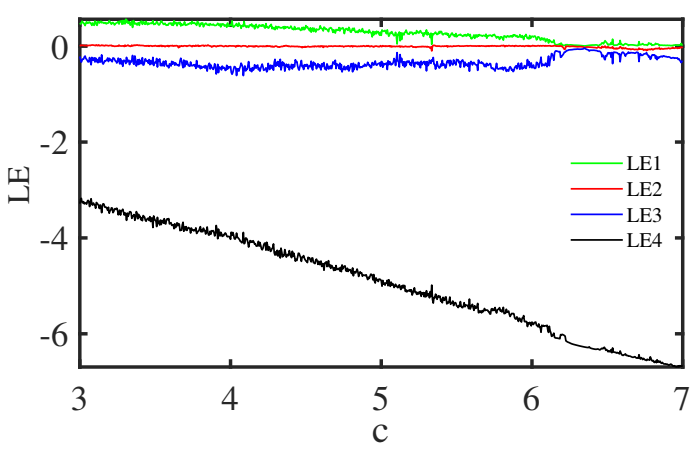

(a)

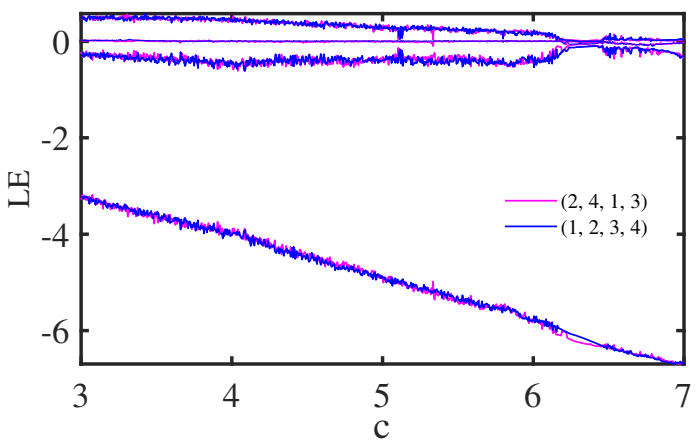

(c)

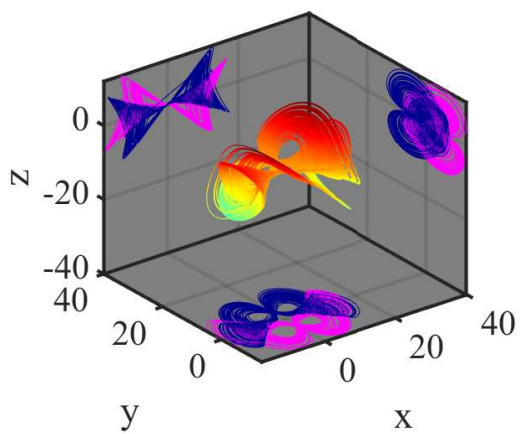

(e)

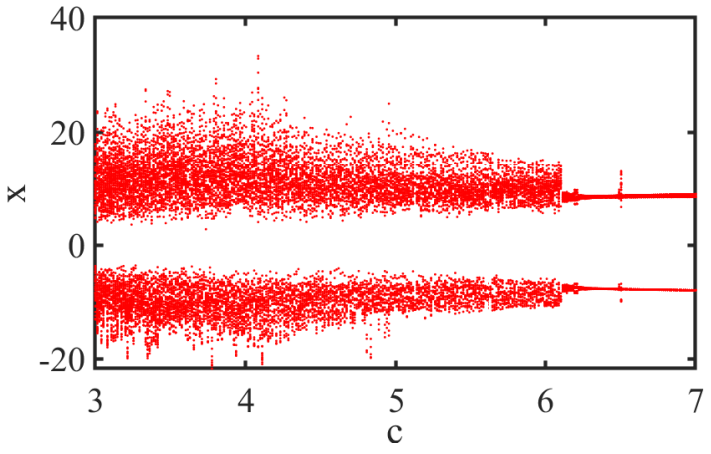

(b)

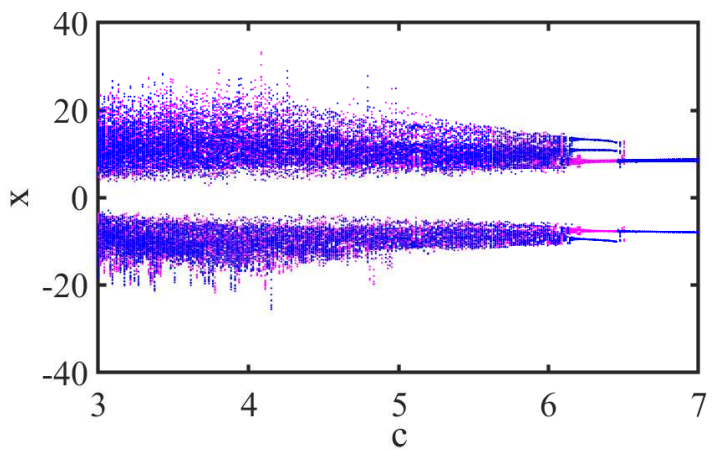

(d)

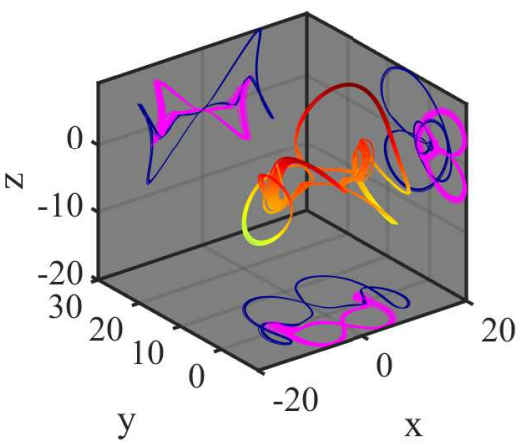

(f)

Fig. 11 Dynamical behaviors varying with parameter $c$ from 3 to 7 with $x_{0}=(2,4,1,3)$ and $x_{1}=(1,2,3,4)$. a Lyapunov exponents; $\mathbf{b}$ bifurcation diagram; c coexisting Lyapunov exponent spectrum; $\mathbf{d}$ coexisting bifurcation diagram; e the coexistence of two chaotic attractors $(c=5.30) ; \mathbf{f}$ the coexistence of periodic and quasi-periodic attractors $(c=6.25)$

Table 3 Circuit parameters corresponding to Fig. 13 where $C_{1}=C_{2}=C_{3}=C_{4}=10 n F, R_{12}=R_{13}=R_{14}=R_{15}=R_{16}=$ $R_{17}=10 k \Omega$.

\begin{tabular}{lll}
\hline System & Closed switches & Resistence value \\
\hline$(2)$ & $S_{1}$ and $S_{6}$ & $R_{1}=R_{8}=R_{11}=1000 k \Omega, R_{2}=R_{4}=R_{6}=R_{7}=100 k \Omega, R_{5}=500 k \Omega, R_{9}=R_{10}=334 k \Omega$ \\
$(3)$ & $S_{3}$ and $S_{4}$ & $R_{1}=R_{2}=R_{4}=R_{6}=R_{7}=R_{8}=R_{11}=100 k \Omega, R_{5}=50 k \Omega, R_{9}=R_{10}=33.4 k \Omega$ \\
$(4)$ & $S_{2}$ and $S_{5}$ & $R_{1}=R_{2}=R_{4}=R_{6}=R_{7}=R_{8}=R_{11}=1000 k \Omega, R_{5}=500 k \Omega, R_{9}=R_{10}=334 k \Omega$ \\
$(5)$ & $S_{4}$ and $S_{5}$ & $R_{1}=R_{2}=R_{4}=R_{6}=R_{7}=R_{8}=R_{11}=1000 k \Omega, R_{5}=500 k \Omega, R_{9}=R_{10}=334 k \Omega$ \\
\hline
\end{tabular}




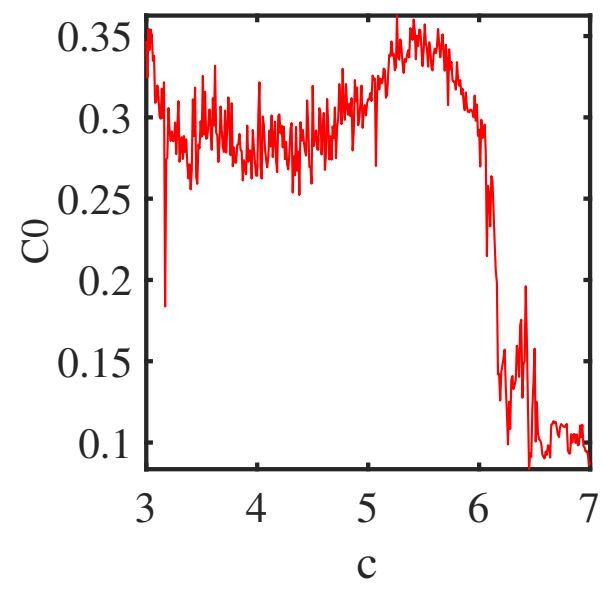

(a)

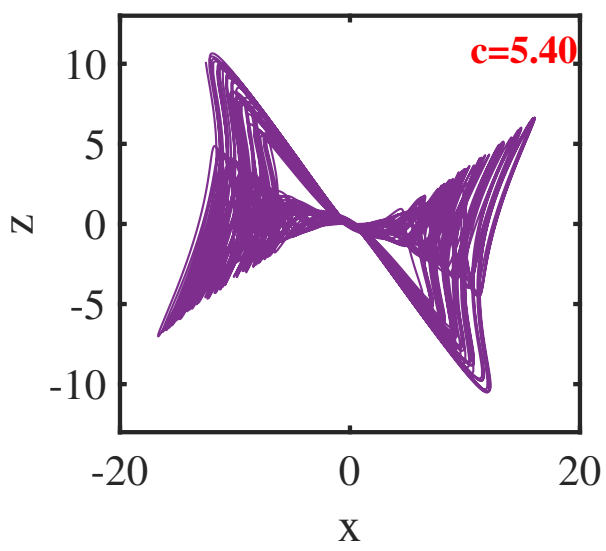

(c)

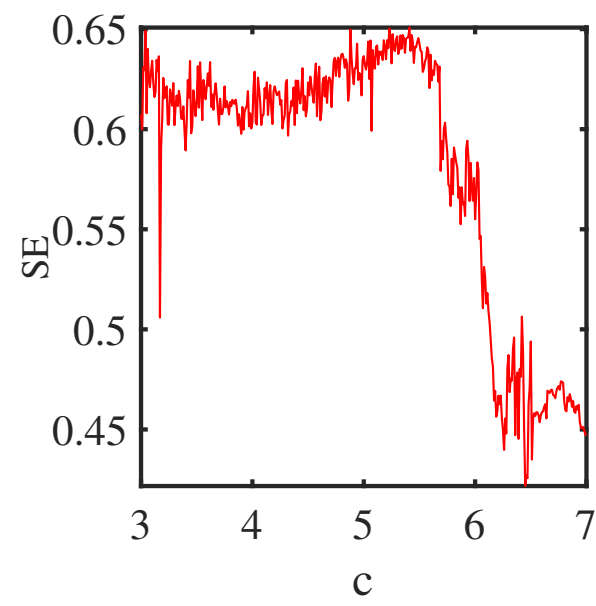

(b)

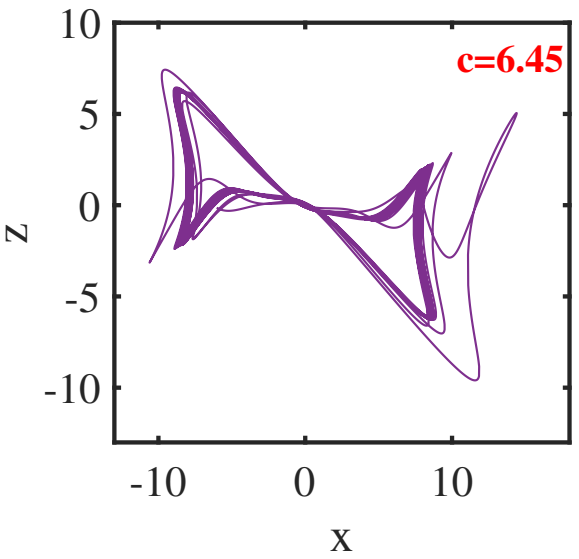

(d)

Fig. 12 The complexity and corresponding attractors portraits of system $(5) x_{0}=(2,4,1,3)$. a $C 0$ complexity; b $S E$ complexity; c $c=5.40 ; \mathbf{d} c=6.45$

\section{Characteristic analysis of fractional-order system (4)}

Compared with the integer-order chaotic system, fractionalorder chaotic system is not only closer to the real world, but also has richer dynamical behaviors and wider applications because of its more complex random consequences [31].

\subsection{System equation}

The most complex system (4) is converted into a fractionalorder chaotic system by adding a differential operator, and its complexity characteristics are studied. The frac- tional mathematical model is (4)

$$
\left\{\begin{aligned}
D^{q} x & =-a x+y z-b z-x y \\
D^{q} y & =2 y-x z \\
D^{q} z & =x y+w-c z \\
D^{q} w & =-3 z-w
\end{aligned}\right.
$$

where $x, y, z$ and $w$ are state variables, $a, b$ and $c$ are constant parameters. According to the calculation method of equilibrium point, the equilibrium points of fractional-order system are the same as that of integer order.

\subsection{Complexity properties}

It is worth mentioning that $C 0$ and $S E$ algorithms are used to measure the complexity of chaotic systems. Fur- 

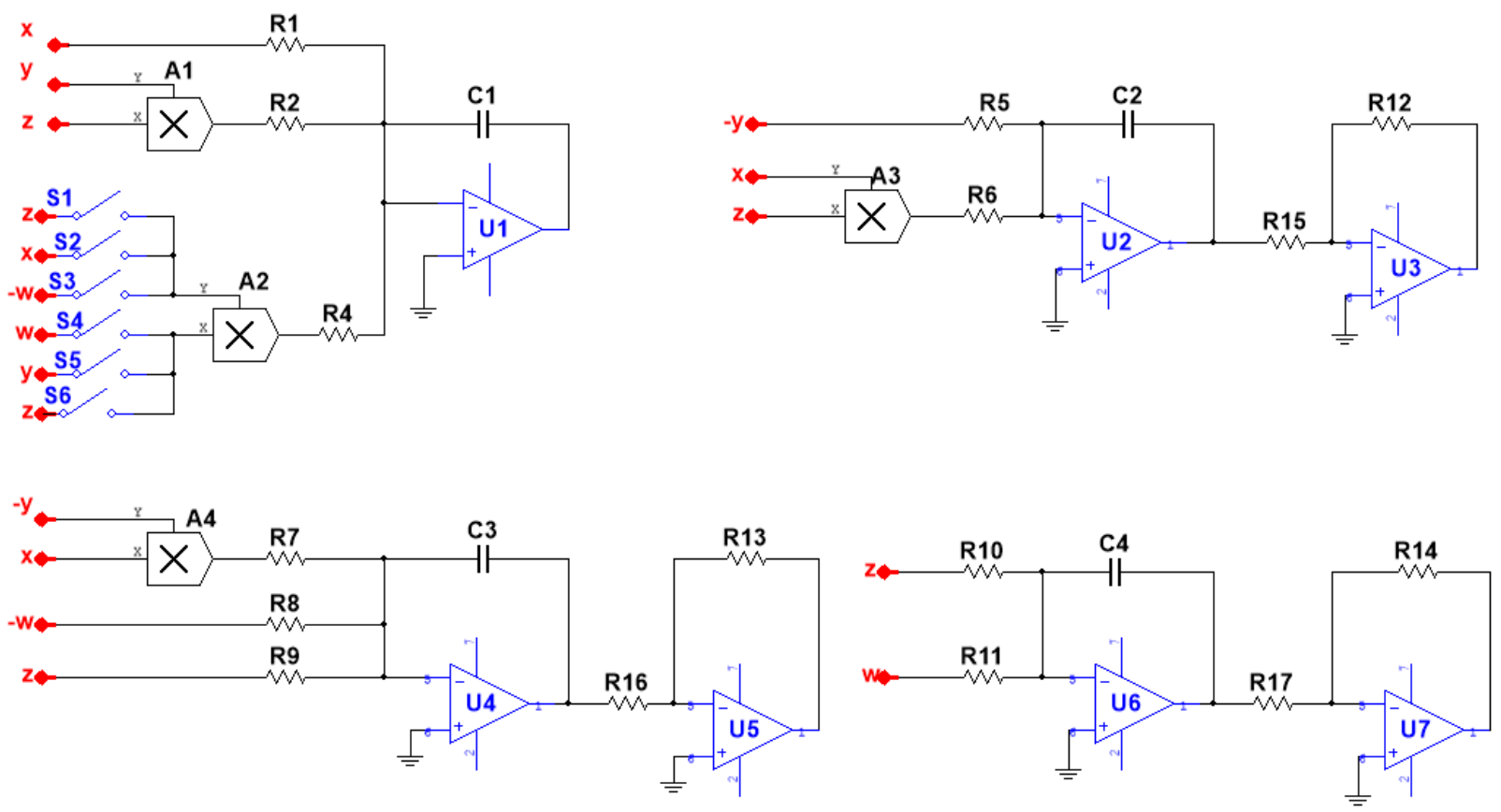

Fig. 13 Circuit schematic diagram of different subsystems implementation.

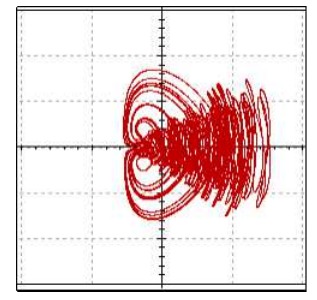

(a)

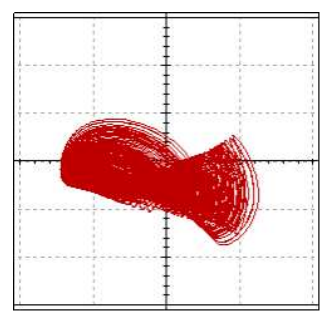

(b)

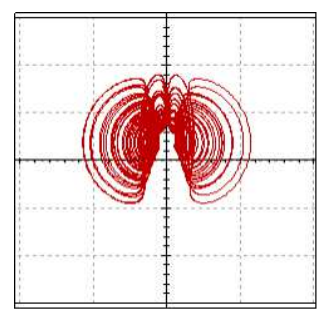

(c)

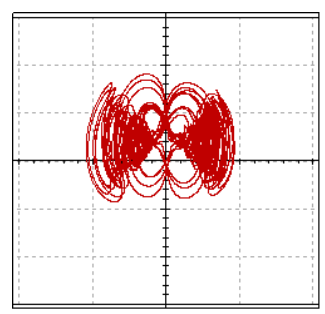

(d)

Fig. 14 Chaotic attractors $\left(V_{x}(\mathrm{~V})-V_{y}(\mathrm{~V})\right)$ of different subsystems obtained from the analog circuit. a system (2); b system (3); c system (4); d system (5)

thermore, the chaos map based on the $C 0$ and $S E$ algorithms can provide a better reference for selecting parameters. The $C 0$ complexity and $S E$ complexity varying with $q \in[0.5,1]$ are shown in Fig. 15. Overall, with the increase of $q$, the complexity value goes down gradually. As you can see from Fig. 16, as parameter $c$ changes, the maximum $C 0$ complexity is approximately 1 and the maximum $S E$ complexity is about 0.7. Come to a conclusion, the complexity of fractional-order system (4) is significantly greater than that of integer order.

While Fig. 17 depicts the variation of the color regions, the darker area represents the maximum complexity of the system. With the color becoming lighter, the complexity of the corresponding system gradually decreases. Actually, it is known that the parameters of low complexity is not advantageous to information security in practical applications [36]. From Fig. 17(a) and (b), the chaotic graphs with an initial value of $(2,4$, $1,3)$ are shown, while (c) and (d) are chaotic maps with an initial value of $(1,2,3,4)$. The results show that the complexity of Fig. $17(\mathrm{c})$ and (d) is more uniform. The complexity values corresponding to different colors are shown in the color bar. In order to select parameters with richer chaotic characteristics, we should consider the parameters of the lower and left area. Combined with Fig. 16 and Fig. 17, the conclusion can be drawn that the parameters are chosen to fractional-order system (4) for $a=1, b=0$, and $c=3$, and , and the initial value $x_{1}=(1,2,3,4)$. The fractional phase diagrams obtained by Adomian algorithm are shown in Fig. 18. The choices of chaos in cryptography and communi- 


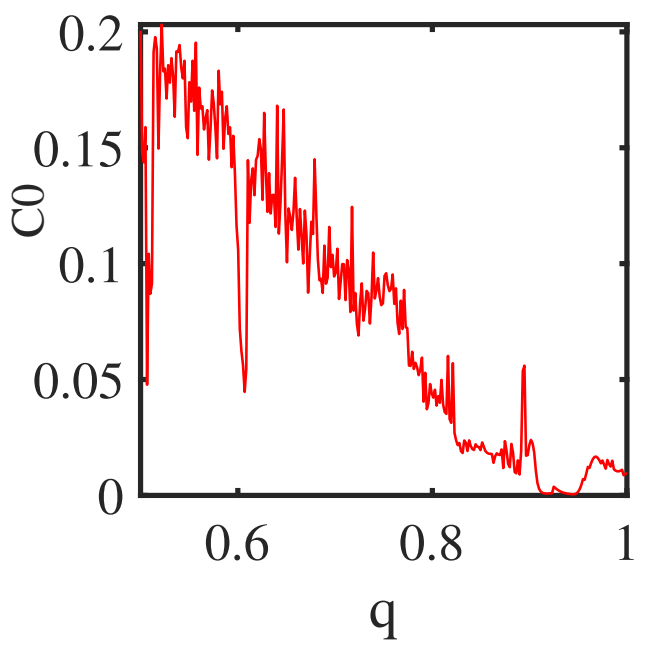

(a)

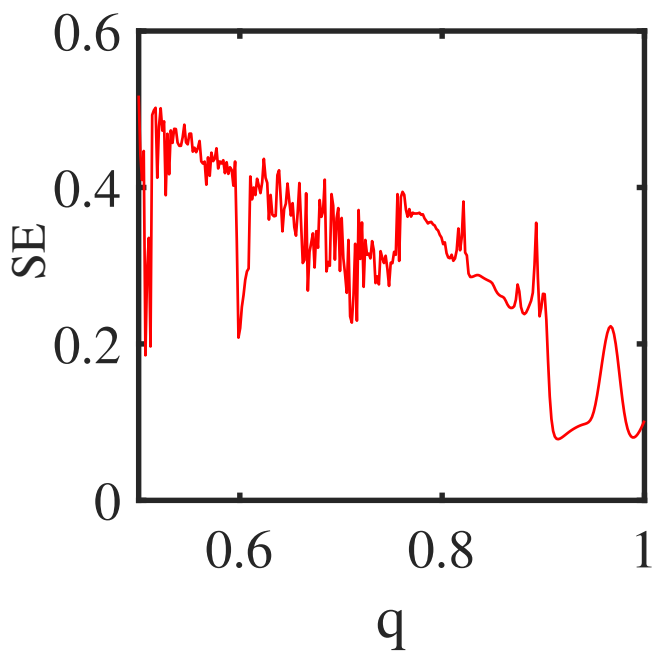

(b)

Fig. 15 Complexity of fractional-order chaotic system (4) varying with $q$. a $C 0$ complexity; b $S E$ complexity

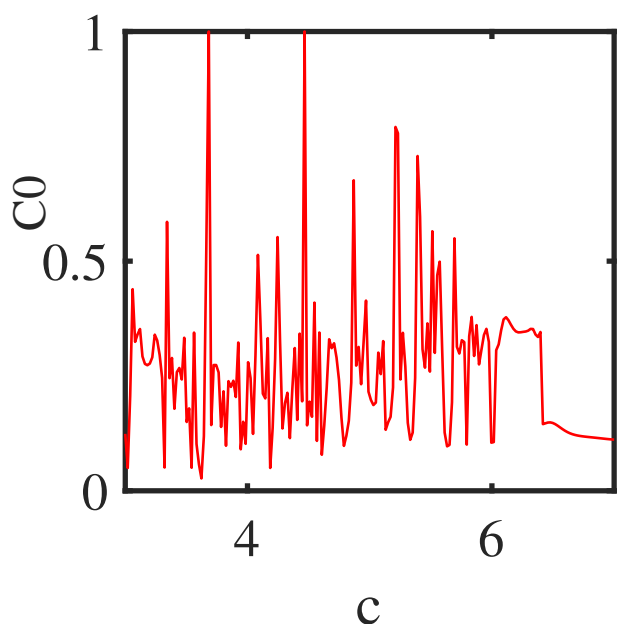

(a)

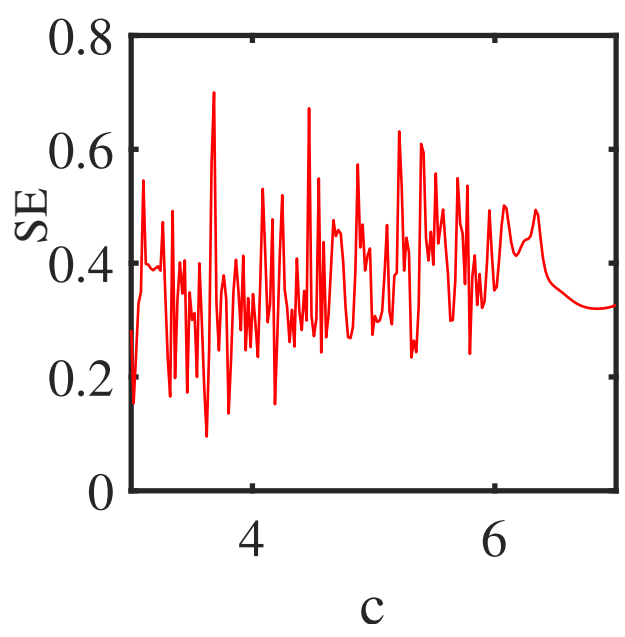

(b)

Fig. 16 Complexity of fractional-order chaotic system (4) varying with $c$. a $C 0$ complexity; b $S E$ complexity.

cations security provide a certain reference, illustrating the practicability of fractional-order chaotic system (4).

\subsection{Circuit Implementation}

In this sub-section, based on the design of integer-order chaotic circuit and fractional-order circuit module, and then replacing integer-order capacitors with fractionalorder modules of order 0.5 , an analog circuit is implemented on Multisim to realize the fractional-order system (4) which can generate the chaotic characteristics in physical sense. The fractional-order circuit module is shown in Fig. 19 and its parameters are shown in Table 4. In detail, the specific circuit parameter values are determined according to the system parameters of the fractional-order system (4). After calculation, they are the same as the circuit parameters of integer-order system. In the same way, fractional-order circuit can also be switchable. 


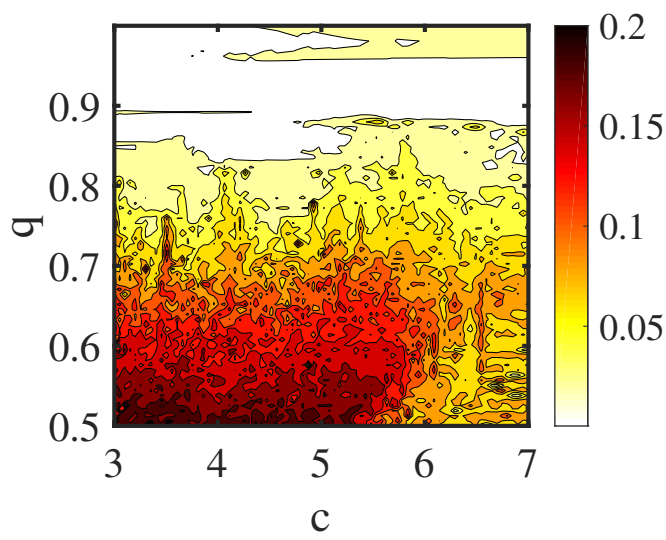

(a)

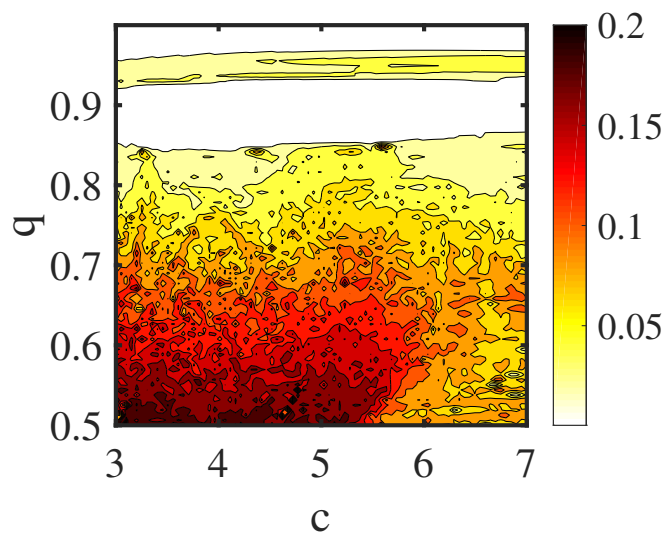

(c)

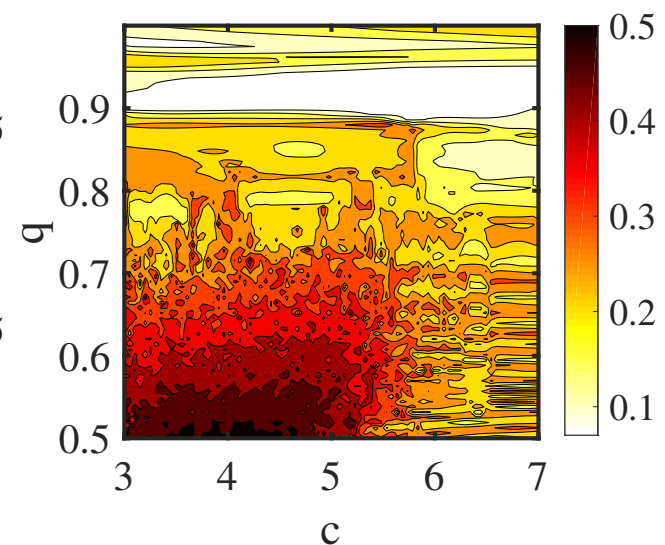

(b)

Fig. 17 Complexity of different initial values of fractional-order system (4) in $c-q$ plane. a the $C 0$ complexity and $\mathbf{b}$ the $S E$ complexity of $x_{0}=(2,4,1,3)$; c the $C 0$ complexity and $\mathbf{d}$ the $S E$ complexity of $x_{1}=(1,2,3,4)$

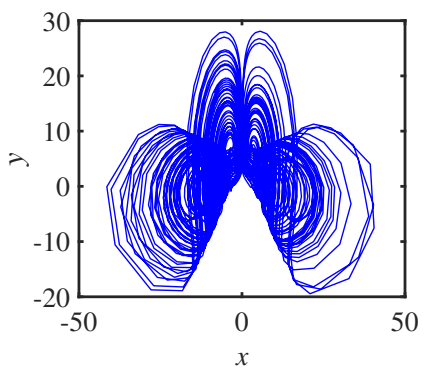

(a)

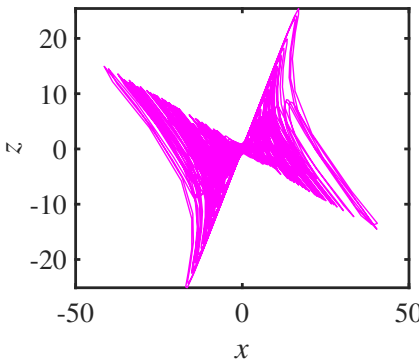

(b)

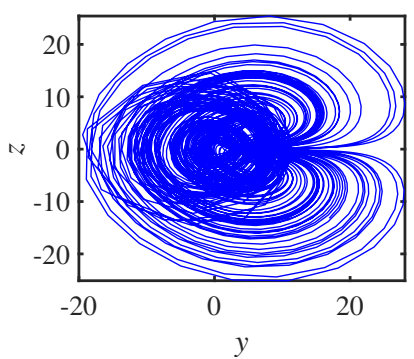

(c)

Fig. 18 Attractor diagrams of fractional-order system (4) with $a=1, b=0$ and $c=3$. a $x-y$ plane; b $x-z$ plane; c $y-z$ plane 


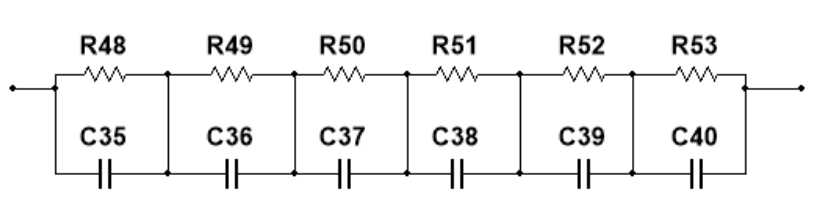

Fig. 19 Fractional-order unit circuit with chaotic order $q=0.5$.

Table 4 Parameter of fractional module

\begin{tabular}{llll}
\hline Capacitance & value & Resistance & values \\
\hline$C_{5}$ & $9.246 \mu \mathrm{F}$ & $R_{18}$ & $6.824 M \Omega$ \\
$C_{6}$ & $5.145 \mu \mathrm{F}$ & $R_{19}$ & $1.944 M \Omega$ \\
$C_{7}$ & $2.129 \mu \mathrm{F}$ & $R_{20}$ & $0.744 M \Omega$ \\
$C_{8}$ & $0.848 \mu \mathrm{F}$ & $R_{21}$ & $0.296 M \Omega$ \\
$C_{9}$ & $0.324 \mu \mathrm{F}$ & $R_{22}$ & $0.123 M \Omega$ \\
$C_{10}$ & $0.925 \mu \mathrm{F}$ & $R_{23}$ & $0.068 M \Omega$ \\
\hline
\end{tabular}

According to [37], the expression of the fractional unit circuit can be written as follows:

$F(s)=\frac{R_{1}}{s R_{1} C_{1}+1}+\frac{R_{2}}{s R_{2} C_{2}+1}+\cdots+\frac{R_{n}}{s R_{n} C_{n}+1}$

The chaotic attractors of the numerical simulation can be observed in Fig. 18. The results of Multisim simulation of fractional-order chaotic circuit are shown in Fig. 20, which correspond to results in Fig. 18, proving that this system is feasible.

\section{FPGA implementation of fractional-order system (4)}

The digital devices on FPGA have attracted the attention of many researchers. Moreover, it is easy to generate chaotic signals with the characteristics of high-speed operation, high integration, free design and so on [38]. An experimental circuit based on FPGA is adopted to implement fractional-order system (4). According the hardware block diagram in Fig. 21, the chaotic attractor of fractional-order system (4) is observed on an oscilloscope through the transformation of the $\mathrm{D} / \mathrm{A}$ development board. By observing Fig. 22, the phase diagram is given to verify the numerical simulation results, it can be concluded that the chaotic attractor realized by FPGA is in good agreement with the chaotic attractor simulated by numerical simulation.

\section{Conclusion}

A four-dimensional chaotic system is designed in this paper. The complex dynamical behaviors of the chaotic system are verified by Lyapunov exponent spectrum, bifurcation diagram, coexisting LEs and coexisting bifurcation. The most complex integer-order chaotic system (4) was obtained via analyzing and comparing the complexity of the systems, and the switching of various circuits is realized by analog circuit. When the corresponding fractional order $q=0.5$ and parameter $c=3$ are selected, the complexity is greater. In this case, the randomness and security of the chaotic sequence are better. Different from previous studies, the circuit can switch the different attractors and prove the feasibility of the system. Moreover, both $C 0$ and $S E$ complexity algorithms are used to confirm the consistency of the results. Significantly, the realization of FPGA proves that the fractional-order system (4) is feasible. The results are of great reference value for the application of fractional-order chaotic system in secure communication.

Acknowledgement This work was supported by the Natural Science Foundation of Gansu Province(20JR5R A531).

\section{Compliance with ethical standards}

Conflict of interest The authors declare that we have no conflicts of interests about the publication of this paper.

Data availability declarations Data sharing not applicable to this article as no datasets were generated or analysed during the current study.

\section{References}

1. Li, Y., Wang, H., Tian, Y.: Fractional-order adaptive controller for chaotic synchronization and application to a dual-channel secure communication system. Mod. Phys. Lett. B 33, 1950290(2019)

2. Cao, Y.: Chaotic synchronization based on fractional order calculus financial system. Chaos Solitons Fractals 130, 109410(2020)

3. Yamakou, M.E: Chaotic synchronization of memristive neurons: Lyapunov function versus Hamilton function. Nonlinear Dyn. 101, 487-500(2020)

4. Ouyang, X., Luo, Y., Liu, J., Cao, L., Liu, Y.: A color image encryption method based on memristive hyperchaotic system and DNA encryption. Int. J. Mod. Phys. B 34, 2050014(2020)

5. Hu, G., Li, B.: A uniform chaotic system with extended parameter range for image encryption. Nonlinear Dyn. 103, 2819-2840(2021)

6. Yang, Y., Wang, L., Duan, S., Luo, L.: Dynamical analysis and image encryption application of a novel 


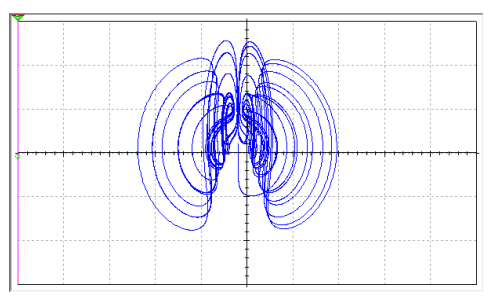

(a)

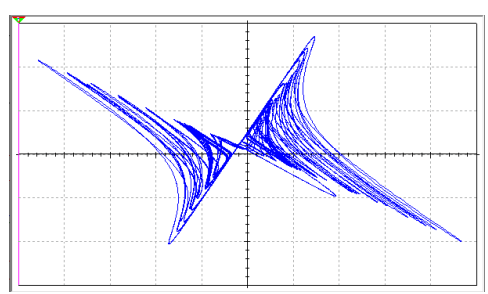

(b)

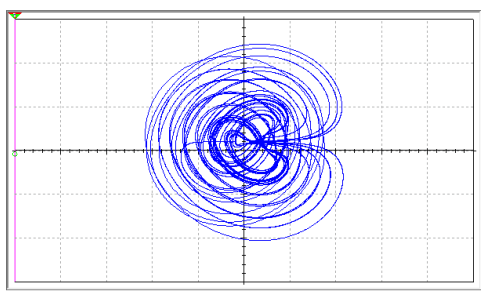

(c)

Fig. 20 Simulation results of fractional-order system. a $x-y$ plane; $\mathbf{b} x-z$ plane; $\mathbf{c} y-z$ pane

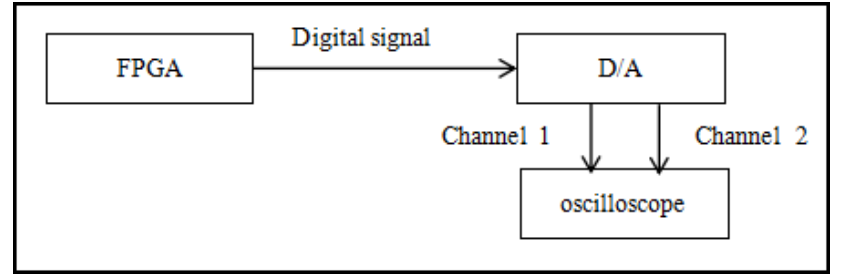

Fig. 21 The hardware block diagram of circuit implementation

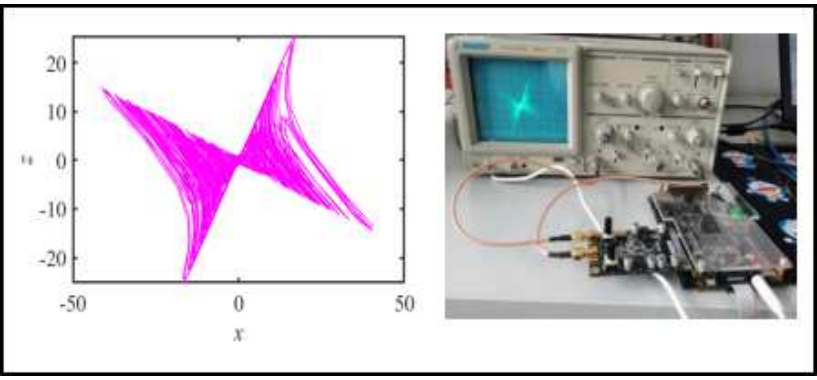

Fig. 22 The results of fractional-order system (4) in the $x-z$ plane. a the numerical simulation; b FPGA

memristive hyperchaotic system. Opt. Laser Technol. 133, 106553(2021)

7. Yang., F., Mou,J., Cao., Y., Chu, R.: An Image Encryption Algorithm Based on BP Neural Network and Hyperchaotic System. China Commun. 17, 2128(2020)

8. Ma, C., Mou, J., Yang, F., Yan, H.: A fractionalorder hopfield neural network chaotic system and its circuit realization. The Eur. Phys. J. Plus 135, 100$100(2020)$

9. Wang, R., Li, C., Iek, S., Rajagopal, K., Zhang, X.: A Memristive Hyperjerk Chaotic System: Amplitude Control, FPGA Design, and Prediction with Artificial Neural Network. Complex. 1, 1-17(2021)

10. Pone, J., Tamba, V. K., Kom, G. H., Mathieu, P., Kom, M.: Numerical, electronic simulations and experimental analysis of a no-equilibrium point chaotic circuit with offset boosting and partial amplitude control. SN Appl. Sci. 1, 922(2019)
11. Fang., S., Li., Z., Zhang, X., Li, Y.: Hidden Extreme Multistability in a Novel No-Equilibrium FractionalOrder Chaotic System and Its Synchronization Control. Brazilian Journal of Physics. Braz. J. Phy. 49, 846-858 (2019)

12. Lai, Q., Kuate, P., Huiqin, P., Fotsin, H.: Infinitely Many Coexisting Attractors in No-Equilibrium Chaotic System. Complex. 21, 1-17(2020)

13. Deng., Q., Wang., C., Yang., L.: Four-Wing Hidden Attractors with One Stable Equilibrium Point. Int. J. Bifurc. Chaos 30, 2050086(2020)

14. Wang, M., Deng, Y., Liao, X., Li, Z., Ma, M., Zeng, Y.: Dynamics and circuit implementation of a fourwing memristive chaotic system with attractor rotation. Int. J. Non-linear Mech. 111, 149-159(2019)

15. Wang, F., Xiao, Y.: A Multiscroll Chaotic Attractors with Arrangement of Saddle-Shapes and Its Field Programmable Gate Array (FPGA) Implementation. Complex. 4, 1-8(2020)

16. Mathale, D., Doungmo Goufo, E. F., Khumalo, M.: Coexistence of multi-scroll chaotic attractors for fractional systems with exponential law and non-singular kernel. Chaos Solitons Fractals 139, 110021(2020)

17. Liu, S., Wei., Y., Liu, J., Chen., S., Zhang, G.: Multi-Scroll Chaotic System Model and Its Cryptographic Application. Int. J. Bifurc. Chaos 28, 1830033(2018)

18. Zhou, W., Wang, G., Shen, Y., Yuan, F., Yu, S.: Hidden Coexisting Attractors in a Chaotic System Without Equilibrium Point. Int. J. Bifurc. Chaos 28, 1830033(2018)

19. Ma, C., Jun, M., Cao, Y., Liu, T., Wang, J.: Multistability analysis of a conformable fractional-order chaotic system. Phys. Scr. 95, 075204(2020)

20. Wang, N., Zhang, G., Ren, L., Bao, H.: Coexisting asymmetric behavior and free control in a simple 3D chaotic system. Int. J. Electron. Commun.(AEÜ) 122, 153234(2020)

21. Lai, Q., Xu, G., Pei, H.: Analysis and control of multiple attractors in Sprott B system. Chaos Soli- 
tons Fractals 123, 192-200(2019)

22. Zhu, F., Wang, F., Ye, L.: Artificial switched chaotic system used as transmitter in chaos-based secure communication. J. Frankl. Inst. 357, 1099711020(2020)

23. Yao, S.: Computing, Design and Implementation of an Automatic Switching Chaotic System between Two Subsystems. Mod. Comput. Sci. Appl. 191, 669-673(2013)

24. Huang, L., Zhang, J., Shi, S.: Circuit simulation on control and synchronization of fractional order switching chaotic system. Math. Comput. Simul. 113, 28-39(2015)

25. Ye, X., Mou, J., Wang, Z., Jin, J., Zhang, L., Liu, E.: Analysis of continuous complexity based on $S E$ and $C_{0}$ algorithm. J. Dalian Polytech. Univ., 2018

26. Ma, C., Mou, J., Xiong, L., Banerjee, S., Liu, T., Han, X.: Dynamical analysis of a new chaotic system: asymmetric multistability, offset boosting control and circuit realization. Nonlinear Dyn. 103, 2867-2880(2021)

27. Li, X., Li, Z., Wen, Z.: One-to-four-wing hyperchaotic fractional-order system and its circuit realization. Circuit World 46, 107-115(2020)

28. Sun, K., He, S., Yi, H., Yin, L.: Complexity analysis of chaotic pseudo-random sequences based on spectral entropy algorithm. Acta Phys. Sin. 62, 709$712(2013)$

29. Chen, H., Lei, T., Lu, S., Dai, W., Qiu, L., Zhong, L.: Dynamics and Complexity Analysis of Fractional-Order Chaotic Systems with Line Equilibrium Based on Adomian Decomposition. Complex. 12, 1-13(2020)

30. Li, G., Zhang, X., Yang, H.: Complexity Analysis and Synchronization Control of Fractional-Order Jafari-Sprott Chaotic System. IEEE Access 99, 11(2020)

31. Wang, L., Sun, K., Peng, Y., He, S.: Chaos and complexity in a fractional-order higher-dimensional multicavity chaotic map. Chaos Solitons Fractals 131, 109488(2020)

32. Chen, J., Yan, D., Duan, S., Wang, L.: Memristorbased hyper-chaotic circuit for image encryption. Chin. Phys. B 29, 299-310(2020)

33. Ye, X., Wang, X., Mou, J., Yan, X., Xian, Y.: Characteristic analysis of the fractional-order hyperchaotic memristive circuit based on the Wien bridge oscillator. Eur. Phys. J. Plus 133, 516(2018)

34. Xian, Y., Mo, Y., Xu, C.: A novel four-dimensional chaotic system with multiple attractor coexisting types. J. South China Univ. Technol. (Nat. Sci. Ed.) 48, 32-43(2020) (in Chinese)
35. Ye, X., Mou, J., Wang, Z.: Complexity analysis of continuous chaotic systems based on SE and C0 algorithms. J. Dalian Polytech. Univ. 37, 71-76(2018)

36. Cui, L., Lu, M., Ou, Q., Duan, H., Luo, W.: Analysis and Circuit Implementation of Fractional Order Multi-wing Hidden Attractors. Chaos Solitons Fractals 138, 109894(2020)

37. Wma, A., Jcs, B.: Chaos in fractional-order autonomous nonlinear systems. Chaos Solitons Fractals 16, 339-3512003(2003)

38. Yu, F., Liu, L., He, B., Huang, Y., Shi, C., Cai, S., Song, Y., Du, S., Wan, Q.: Analysis and FPGA Realization of a Novel 5D Hyperchaotic Four-Wing Memristive System, Active Control Synchronization, and Secure Communication Application. Complex. 12, 1-18(2019) 
Figures

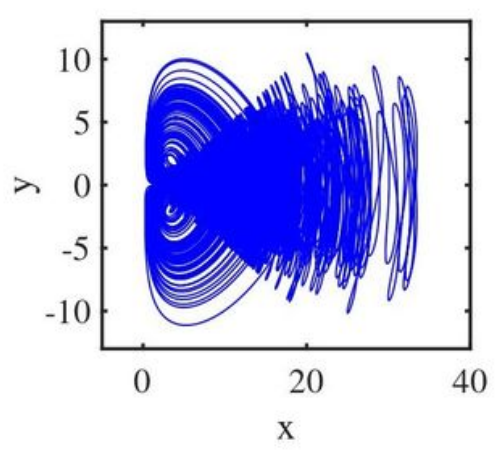

(a)

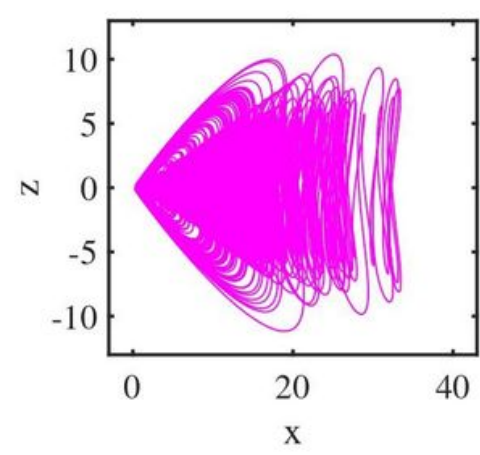

(b)

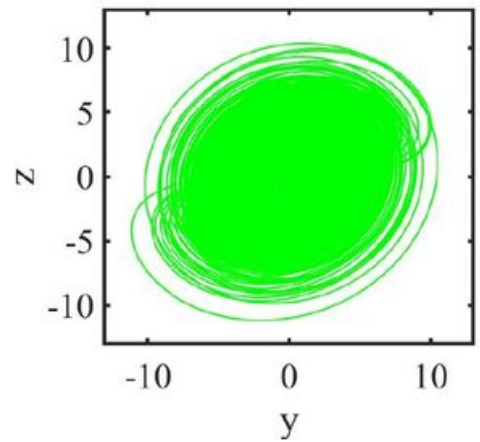

(c)

Figure 1

Please see the Manuscript PDF file for the complete figure caption 


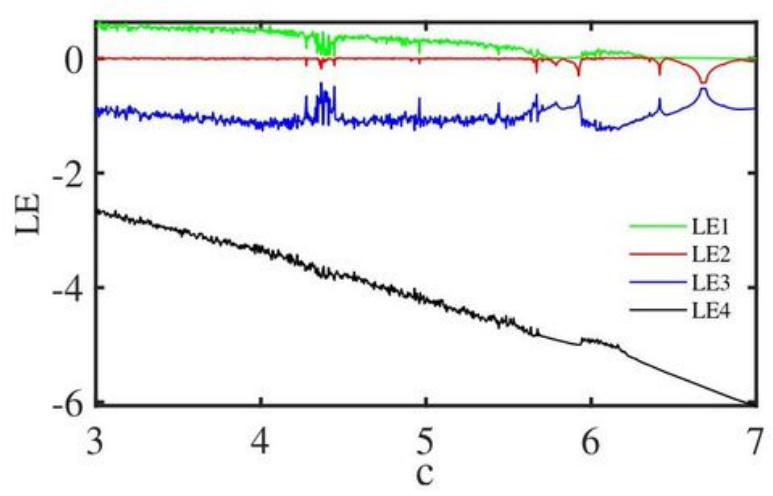

(a)

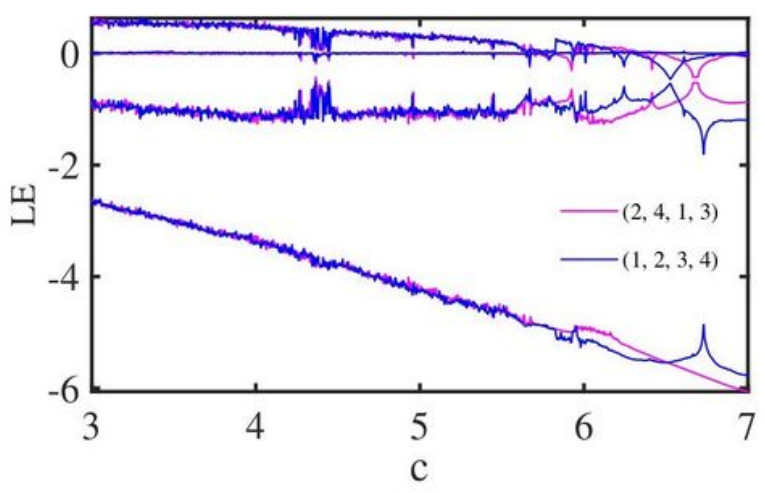

(c)

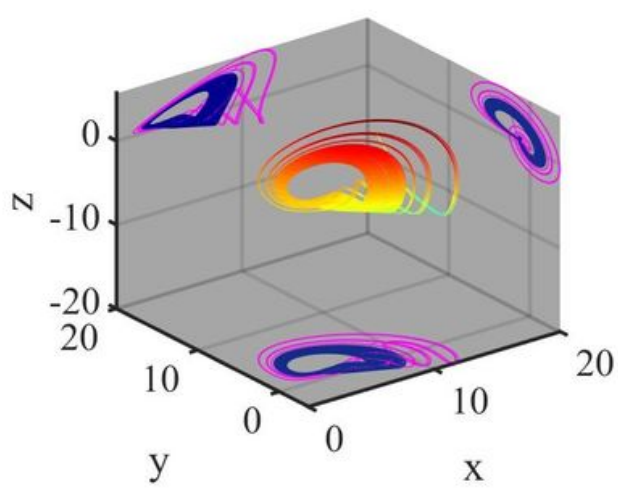

(e)

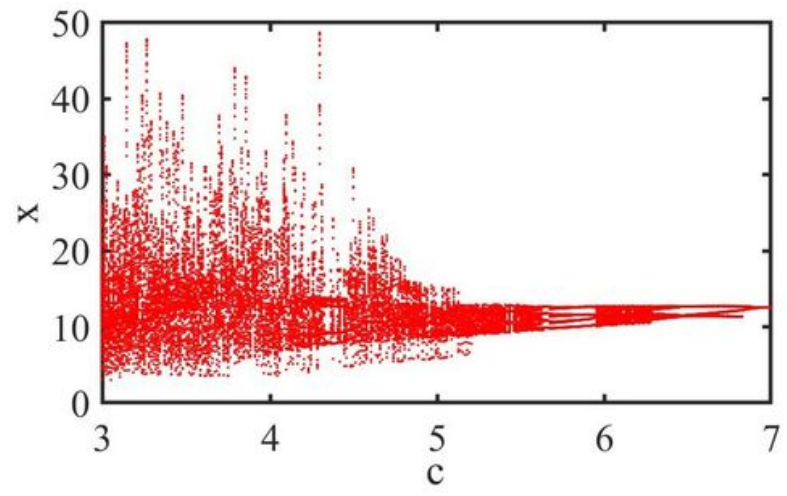

(b)

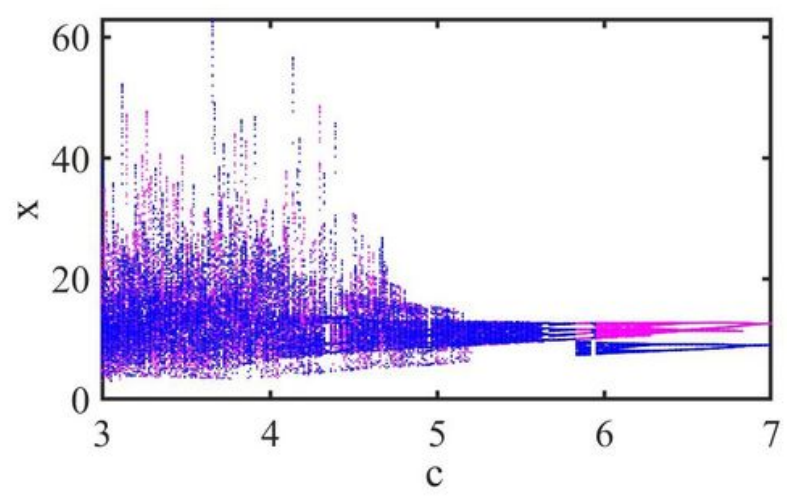

(d)

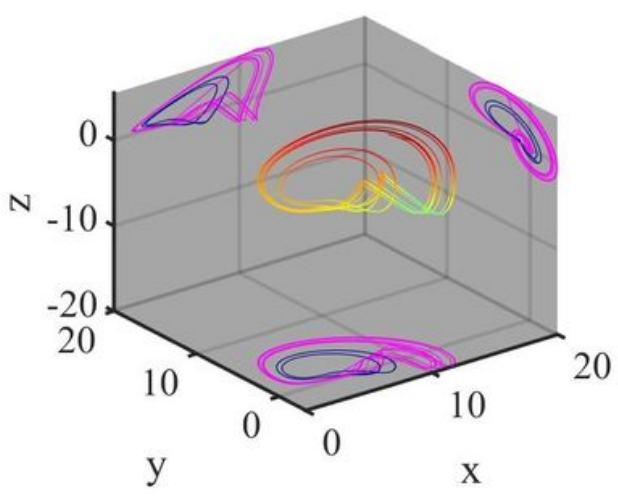

(f)

Figure 2

Please see the Manuscript PDF file for the complete figure caption 


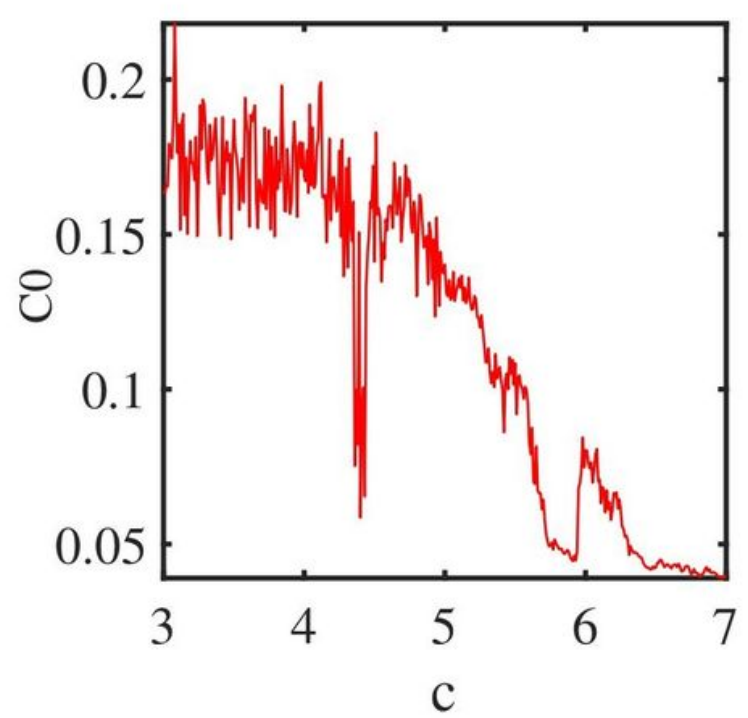

(a)

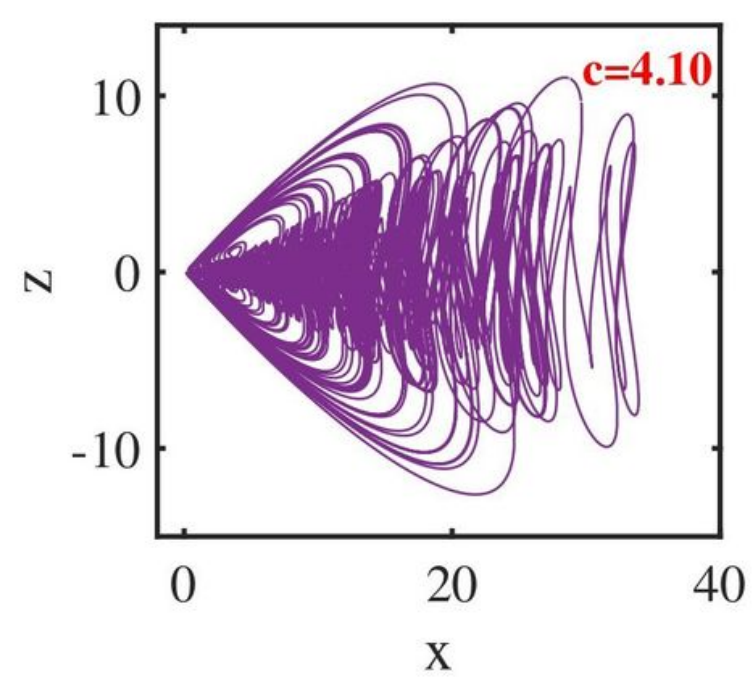

(c)

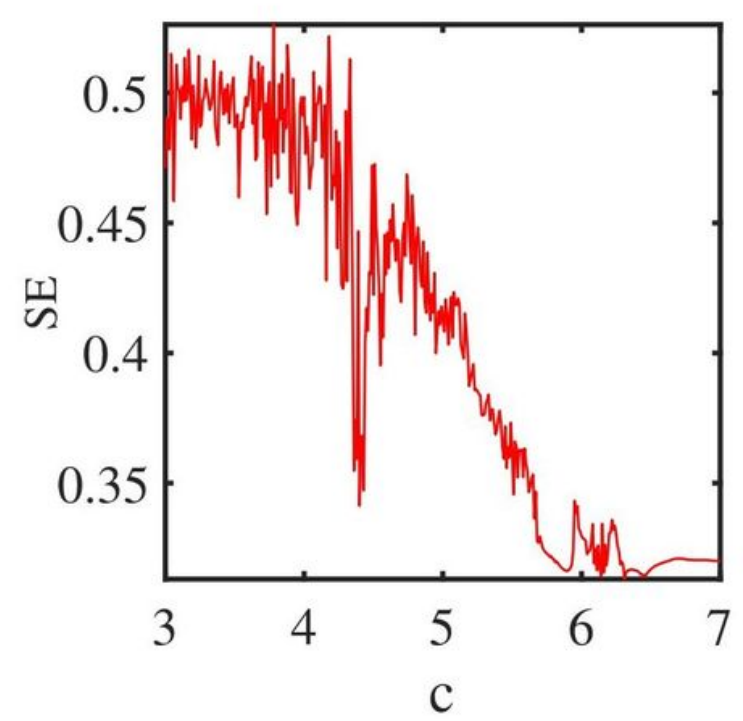

(b)

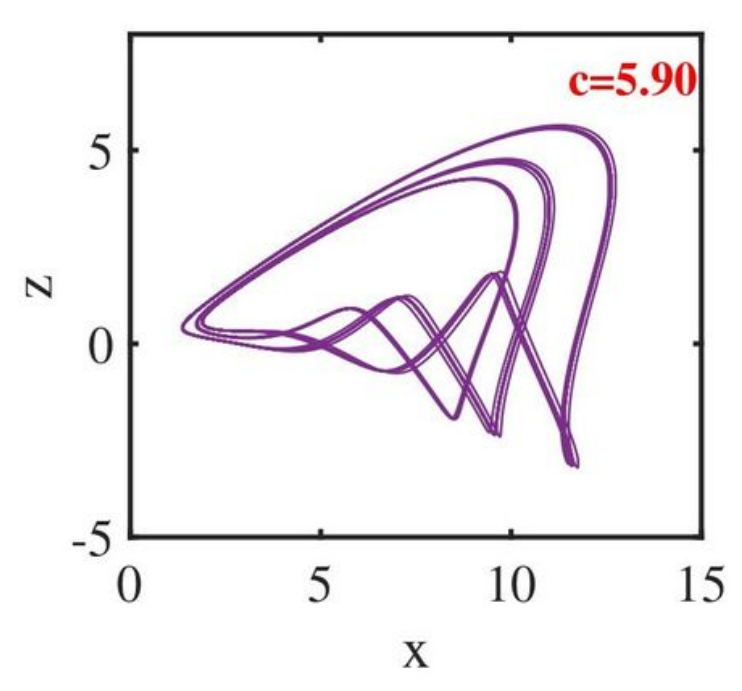

(d)

Figure 3

Please see the Manuscript PDF file for the complete figure caption 


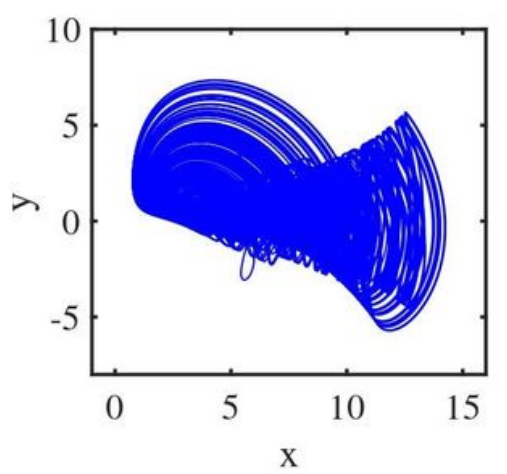

(a)

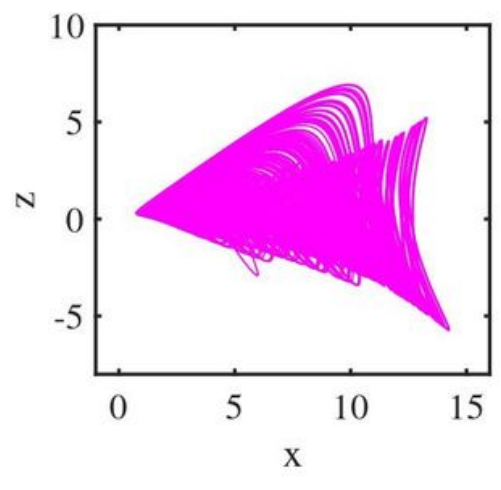

(b)

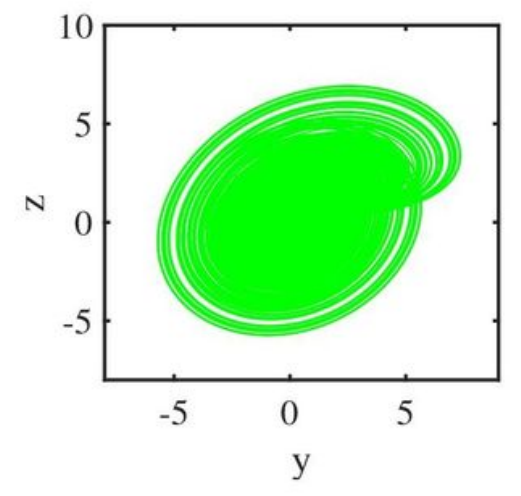

(c)

Figure 4

Please see the Manuscript PDF file for the complete figure caption 


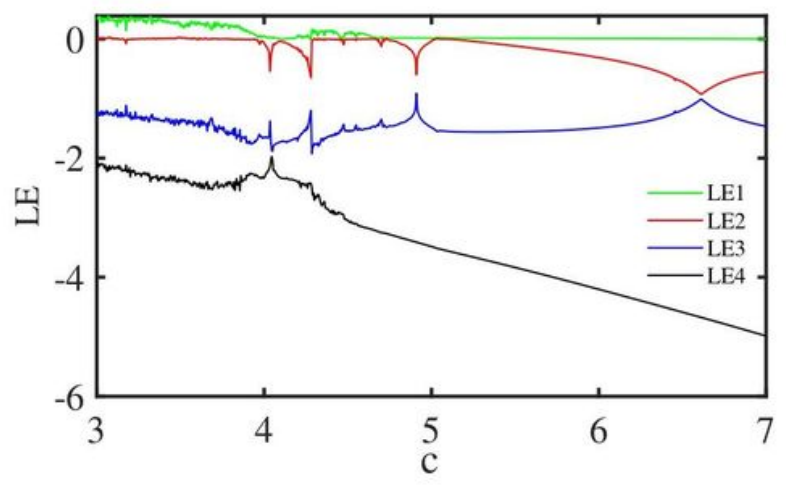

(a)

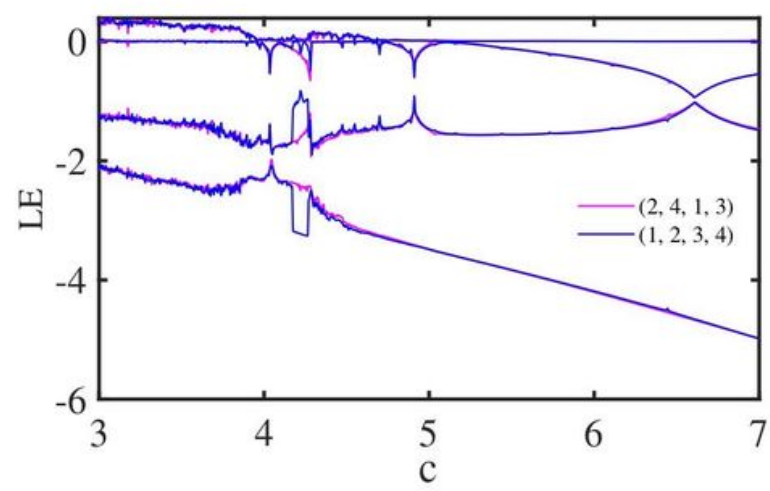

(c)

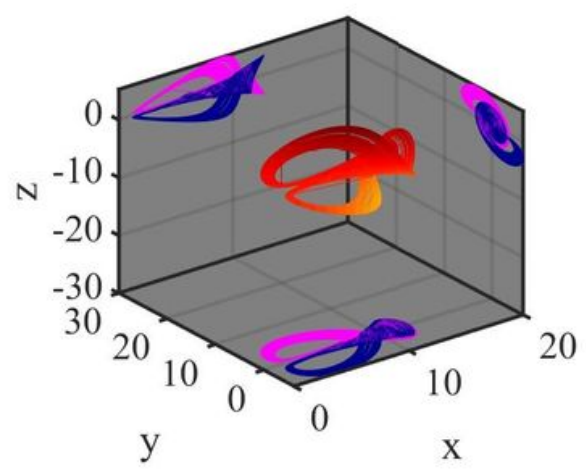

(e)

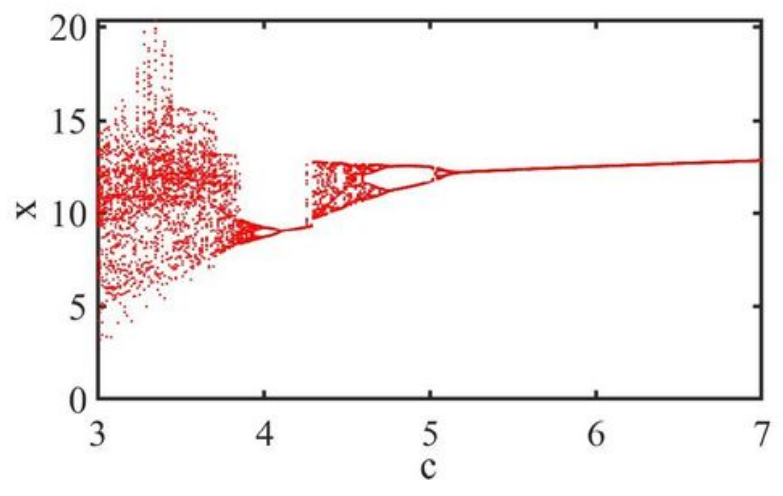

(b)

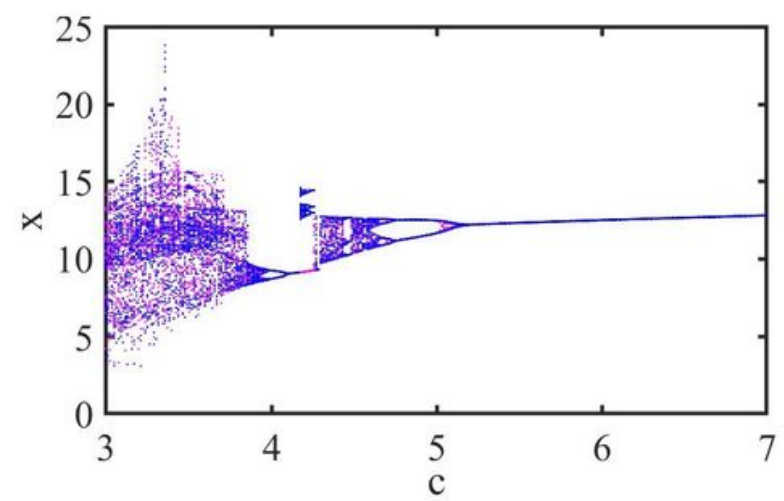

(d)

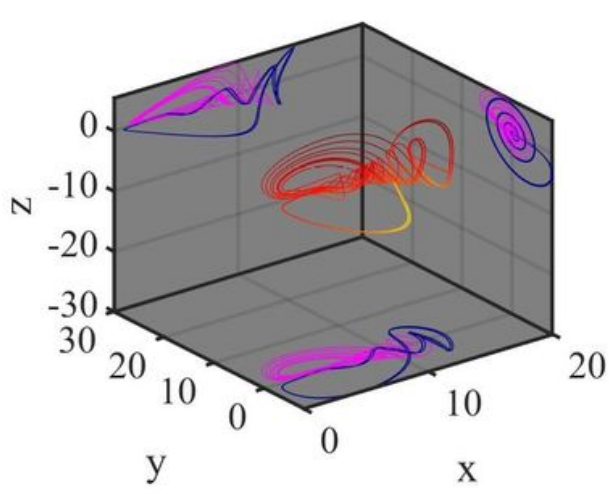

(f)

Figure 5

Please see the Manuscript PDF file for the complete figure caption 


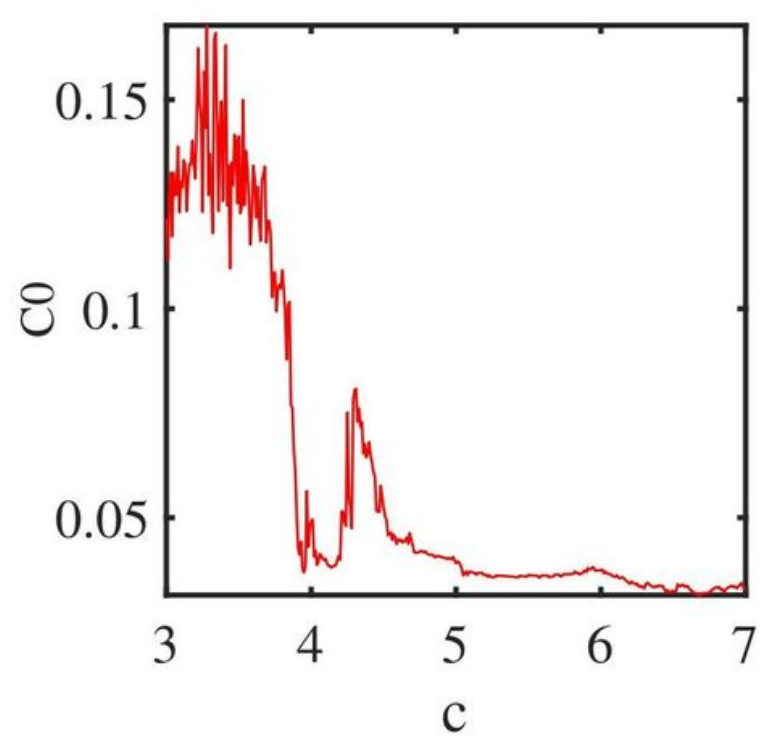

(a)

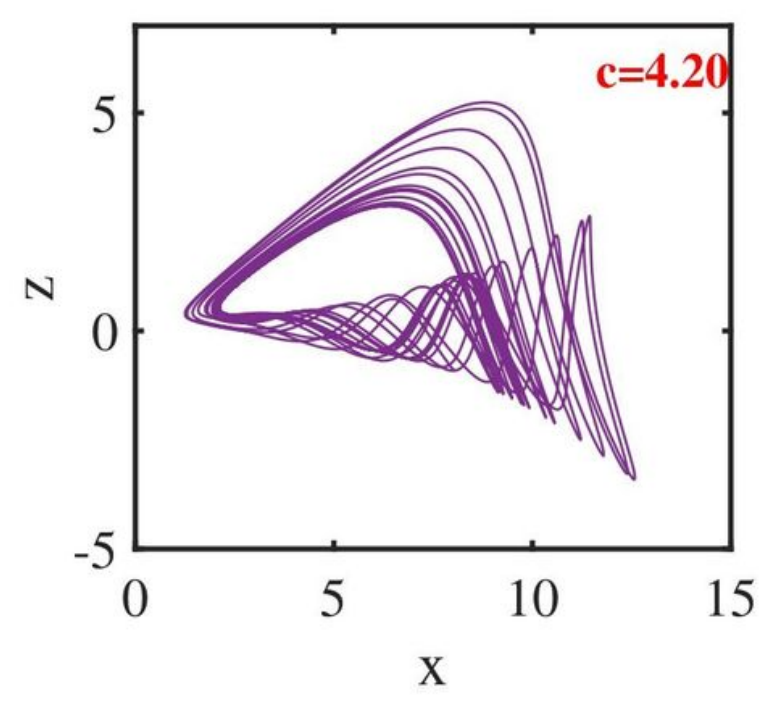

(c)

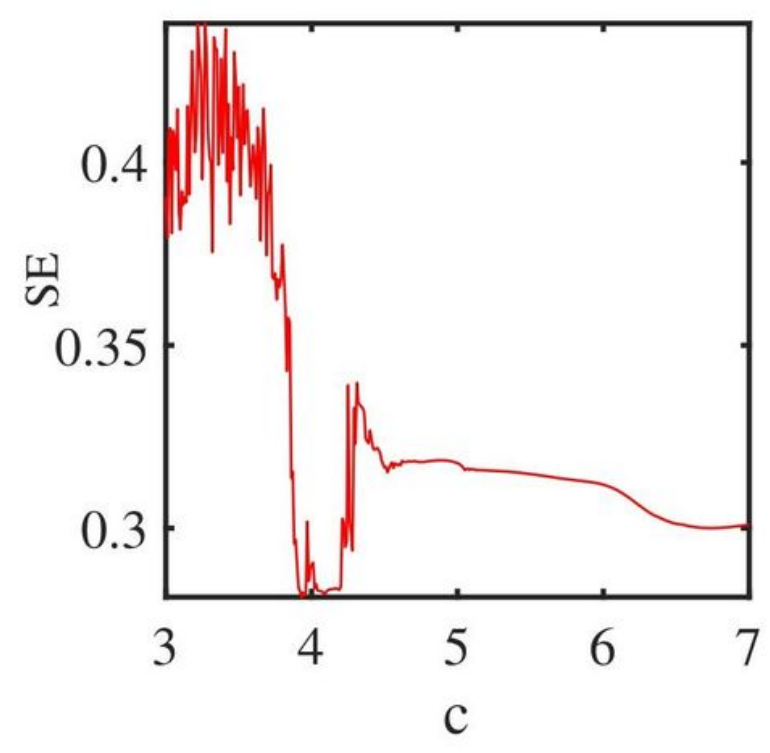

(b)

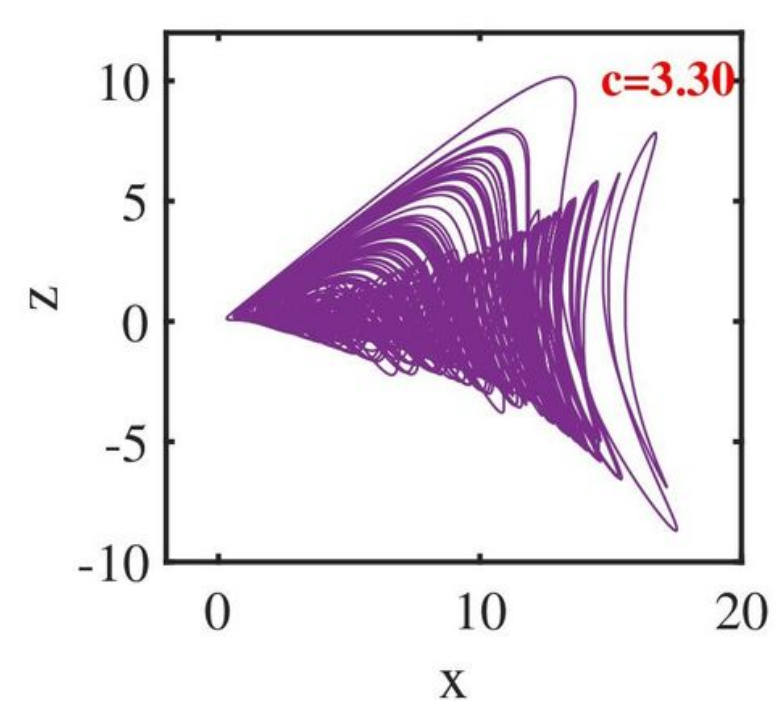

(d)

Figure 6

Please see the Manuscript PDF file for the complete figure caption 


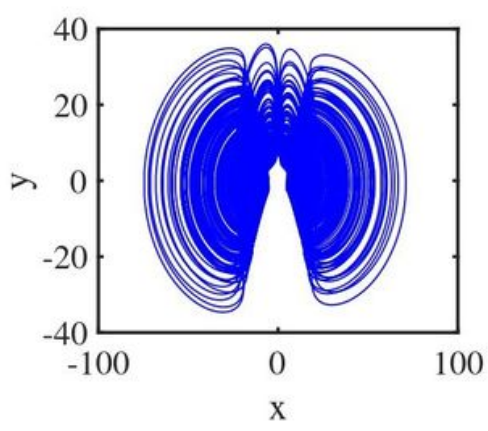

(a)

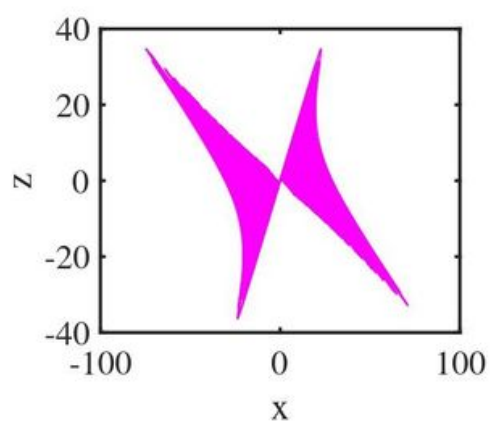

(b)

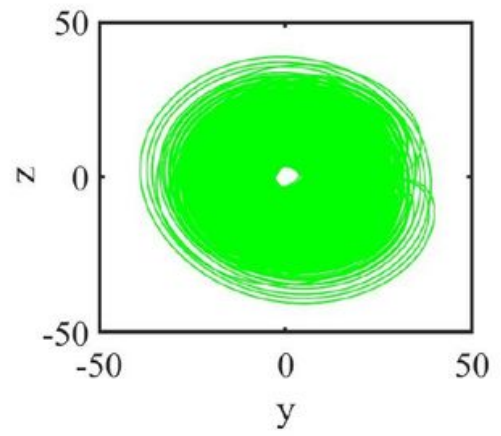

(c)

Figure 7

Please see the Manuscript PDF file for the complete figure caption 


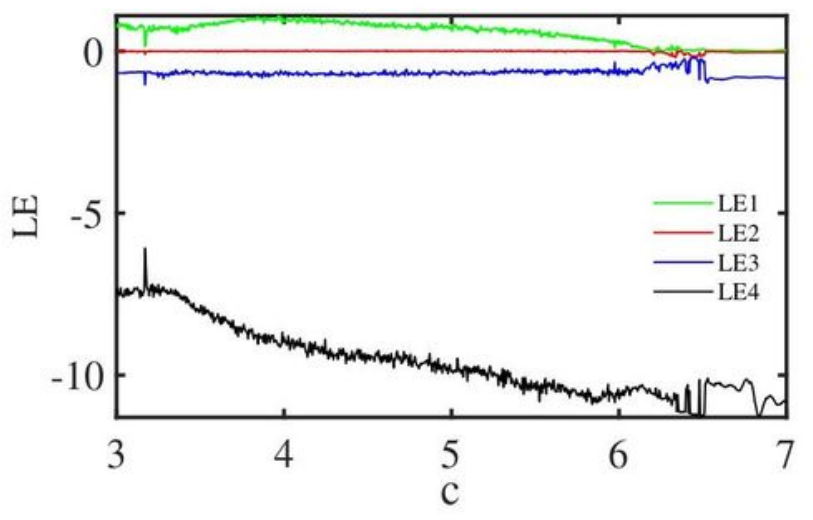

(a)

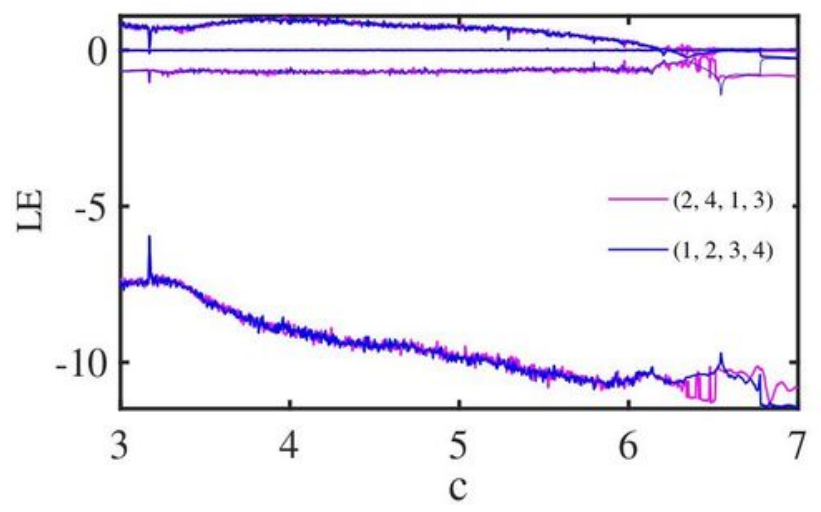

(c)

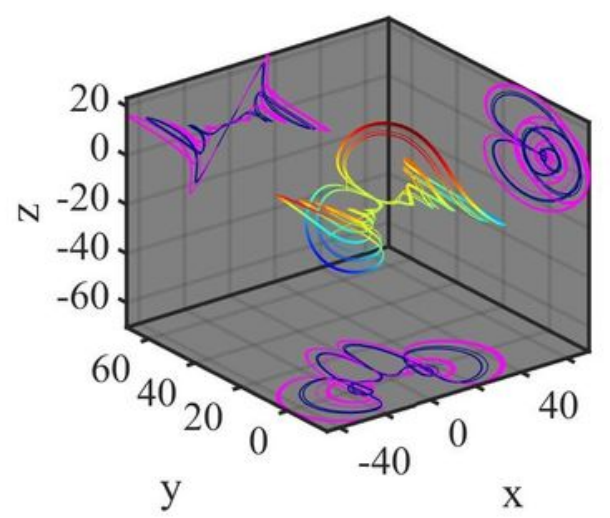

(e)

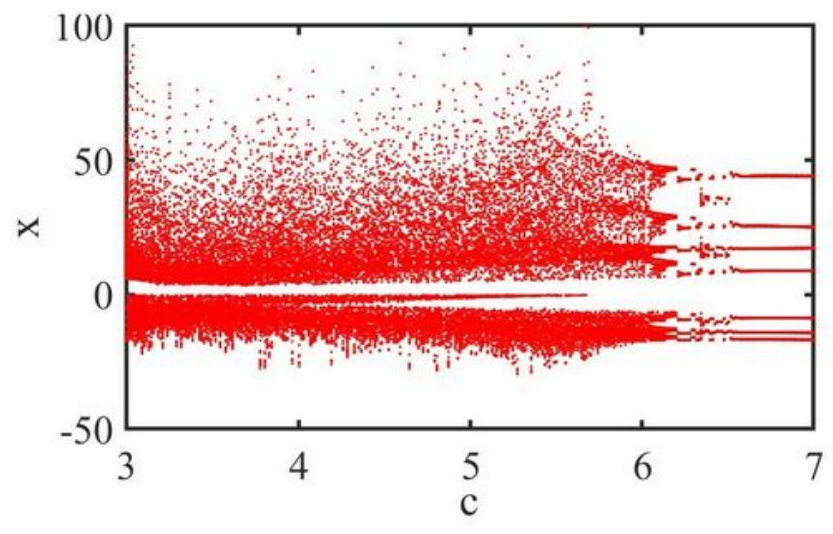

(b)

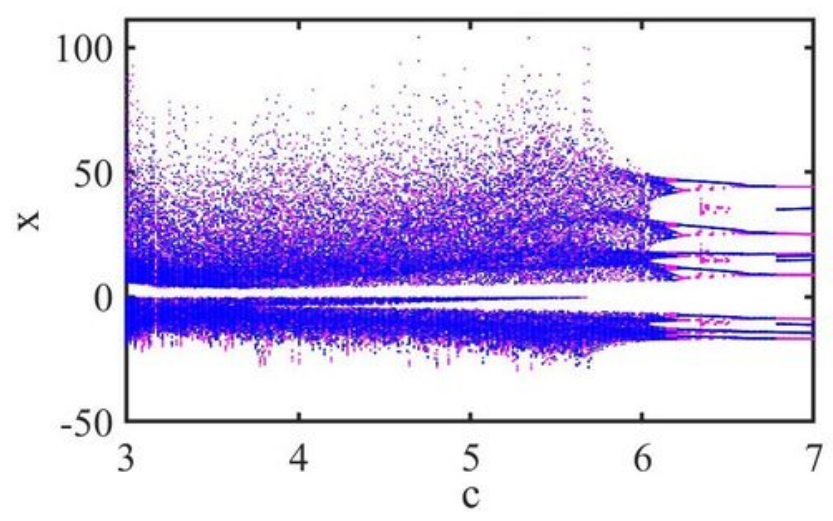

(d)

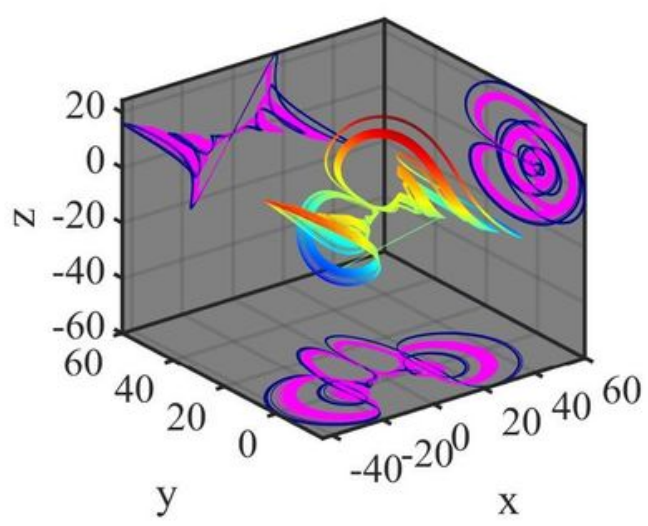

(f)

\section{Figure 8}

Please see the Manuscript PDF file for the complete figure caption 


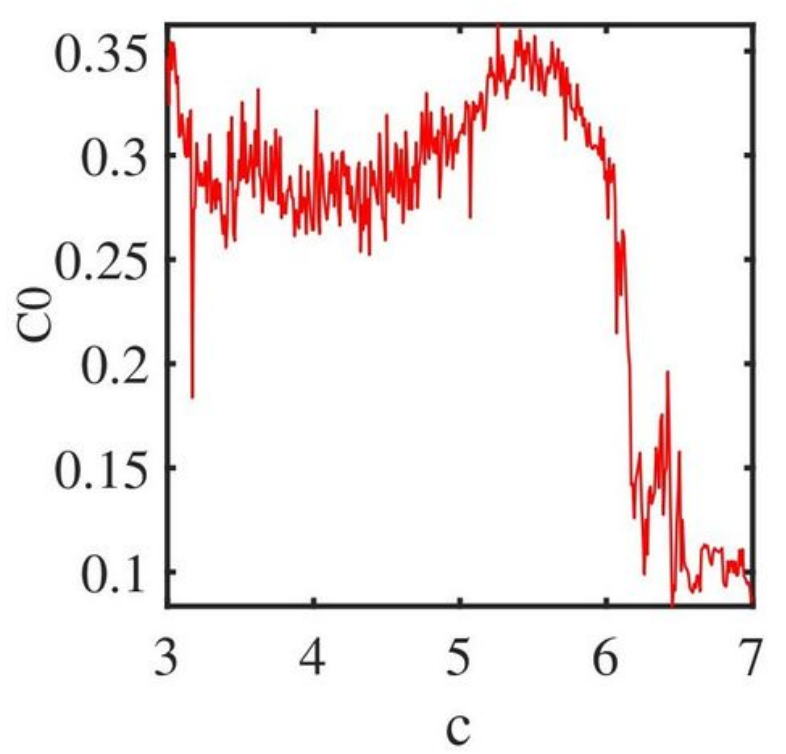

(a)

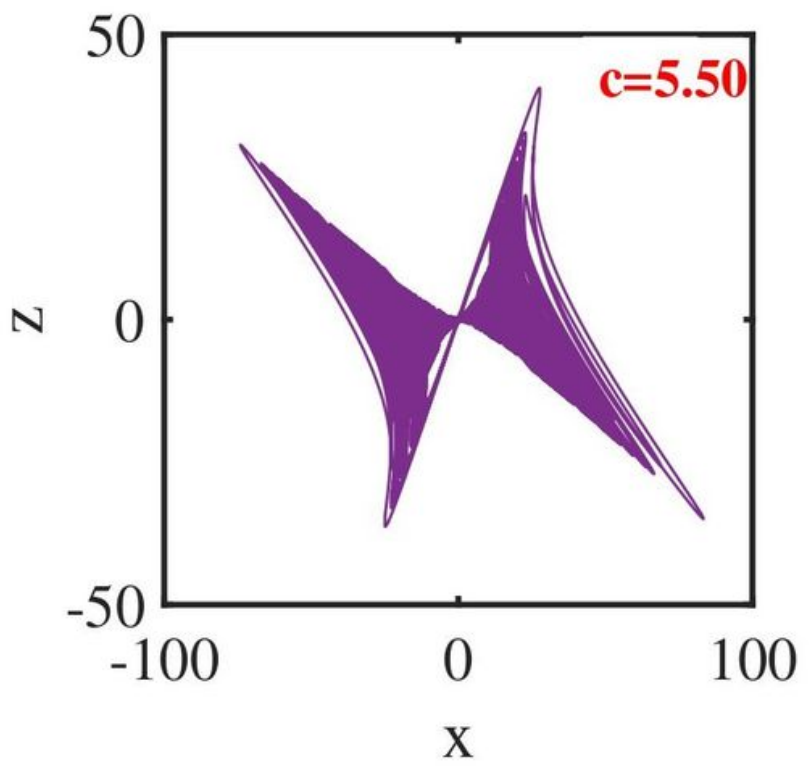

(c)

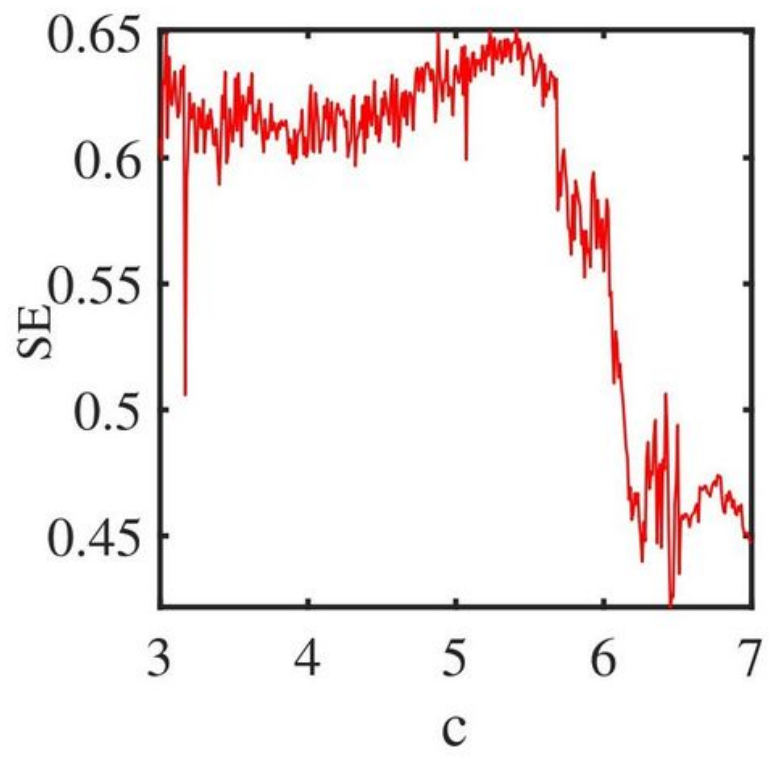

(b)

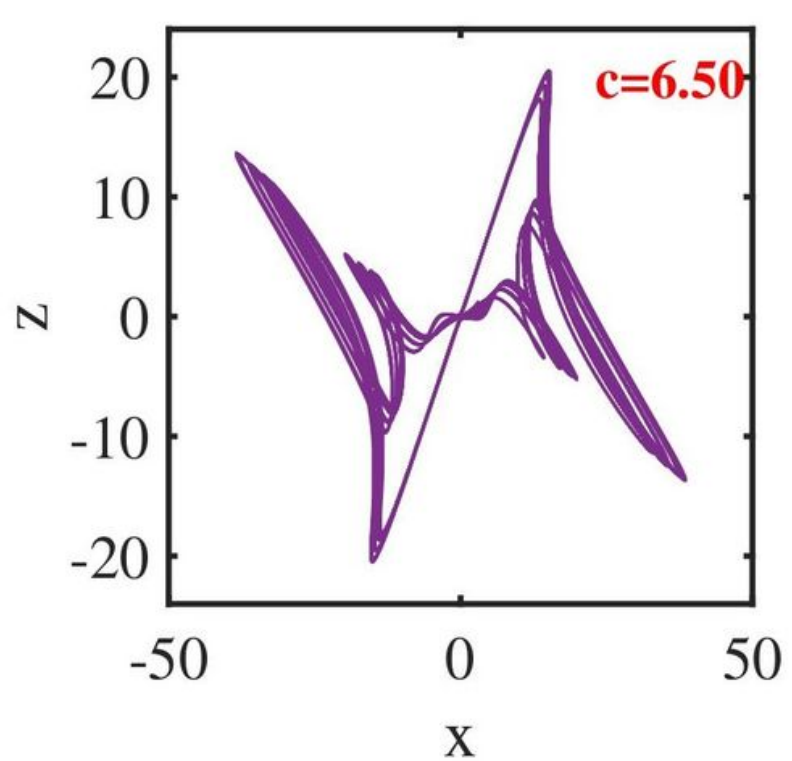

(d)

Figure 9

Please see the Manuscript PDF file for the complete figure caption 


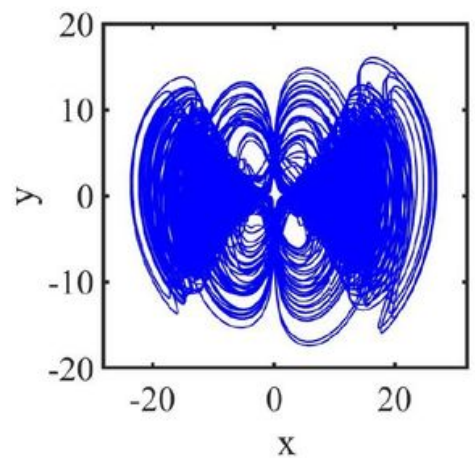

(a)

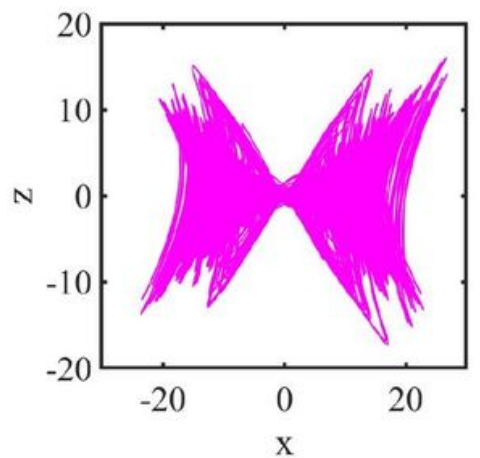

(b)

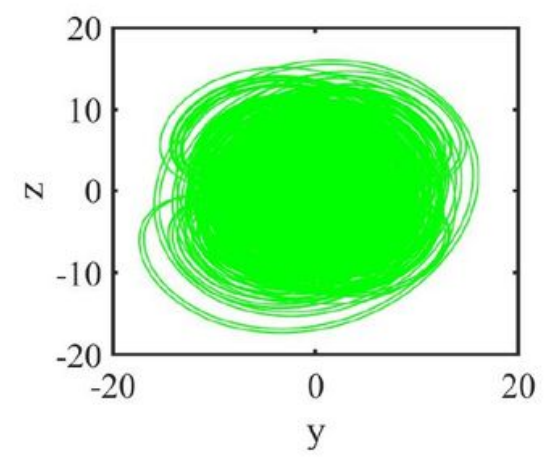

(c)

Figure 10

Please see the Manuscript PDF file for the complete figure caption 


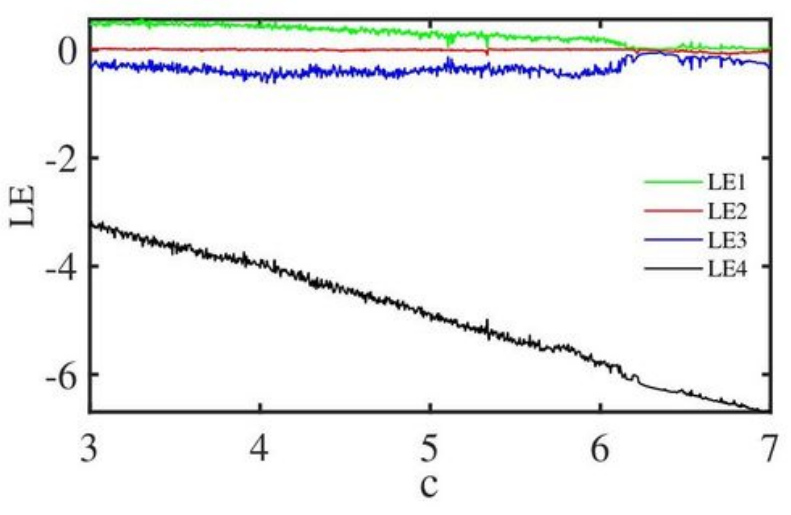

(a)

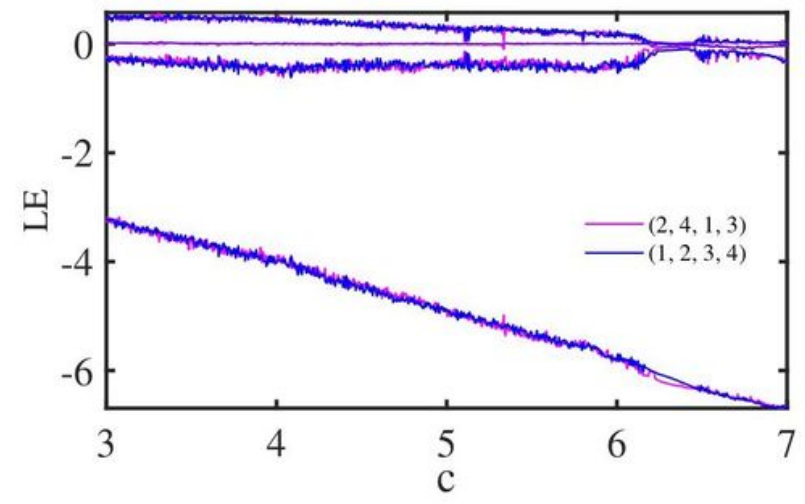

(c)

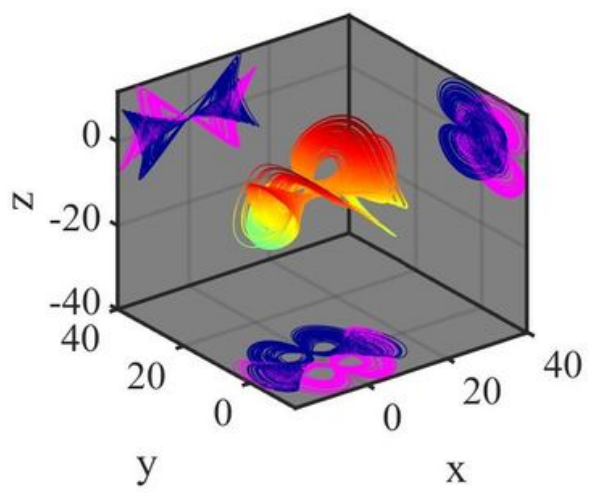

(e)

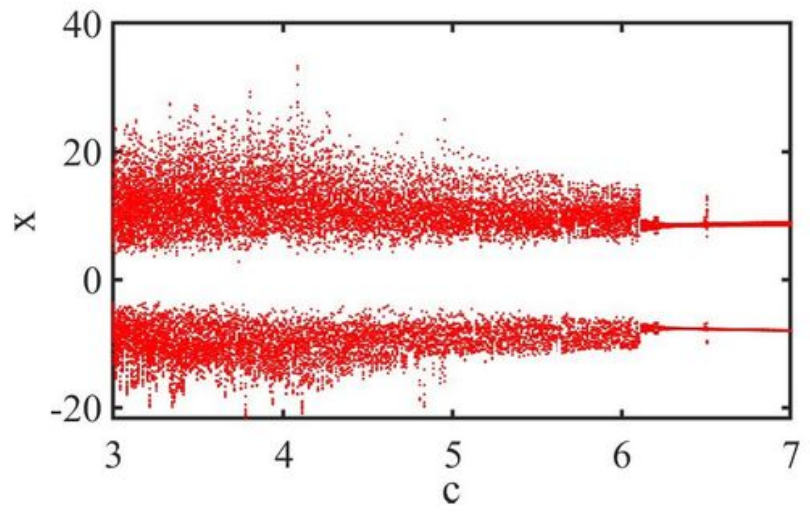

(b)

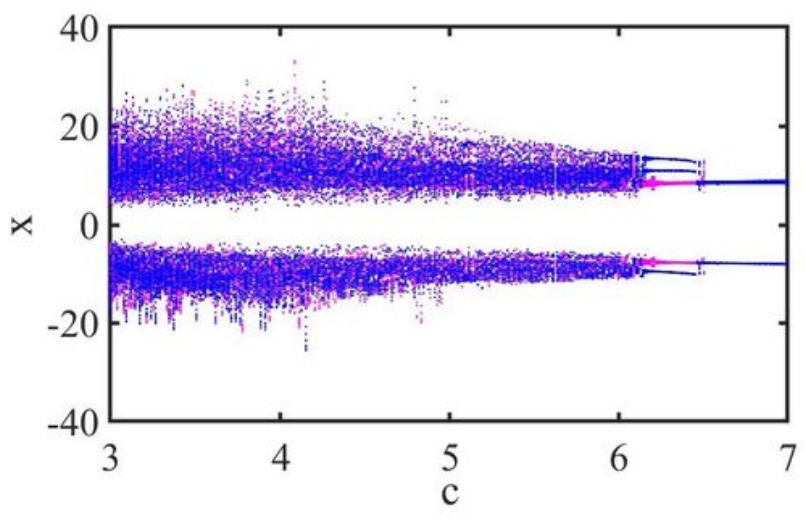

(d)

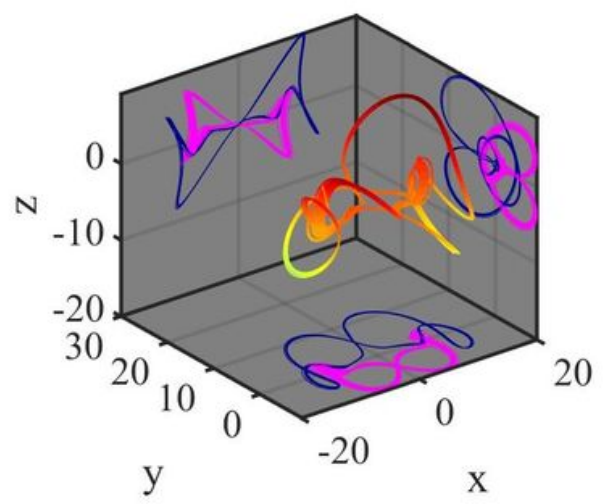

(f)

\section{Figure 11}

Please see the Manuscript PDF file for the complete figure caption 


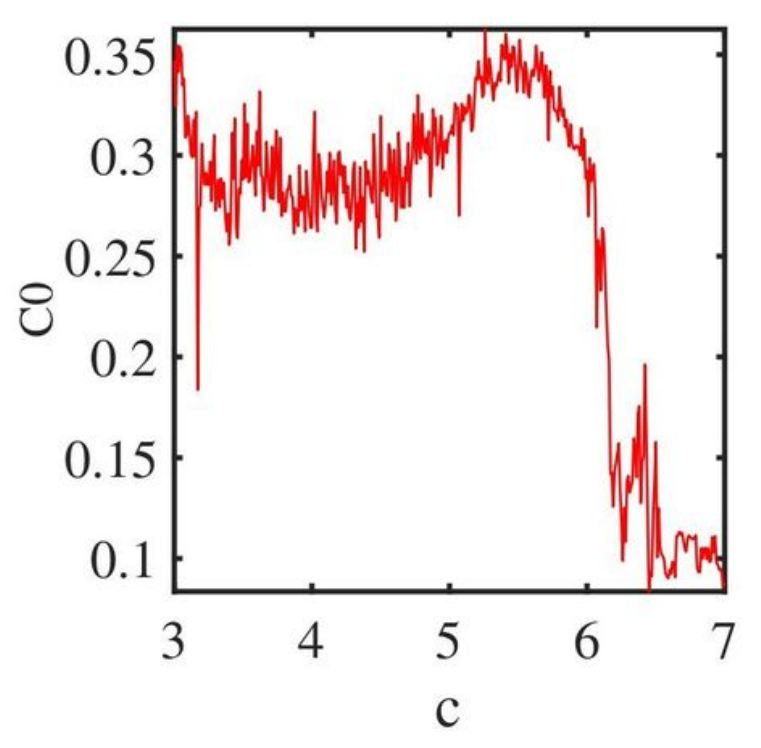

(a)

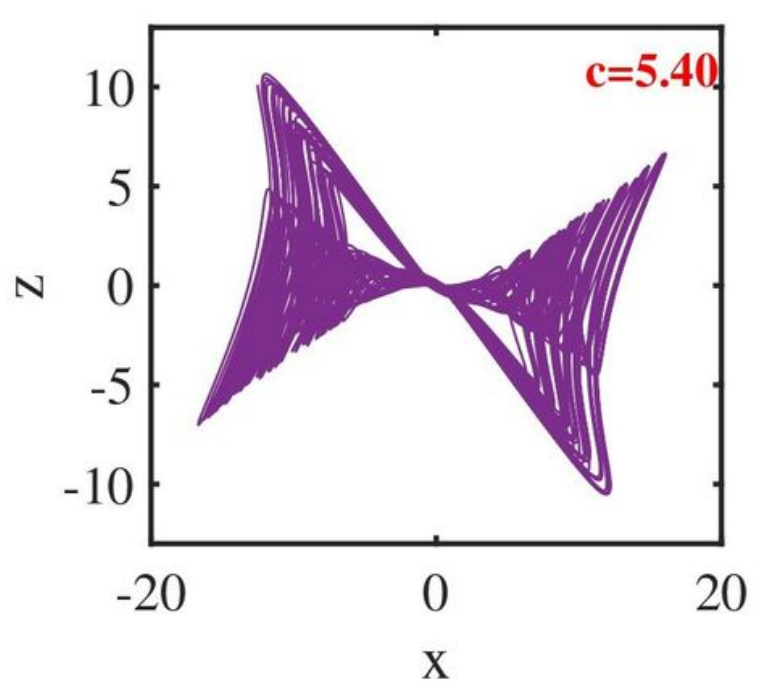

(c)

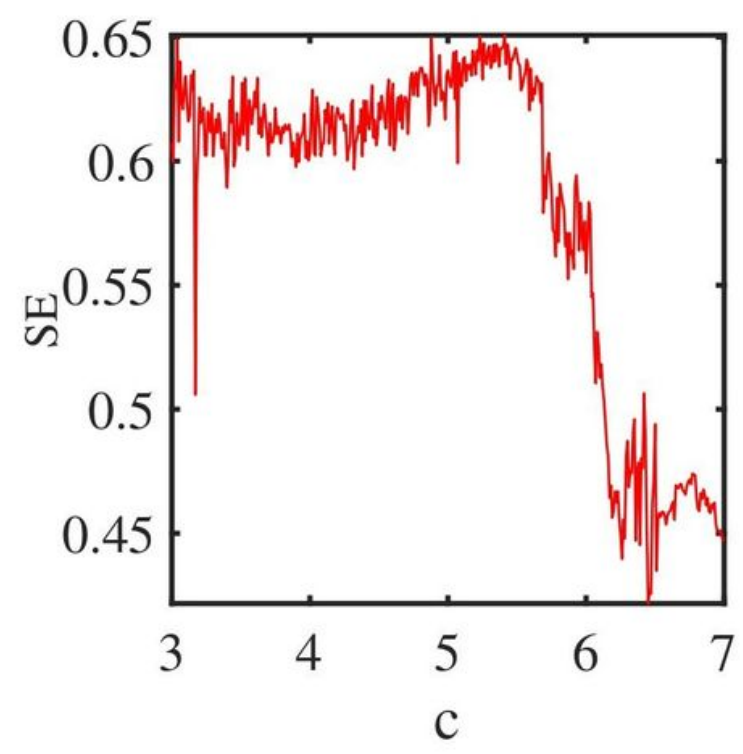

(b)

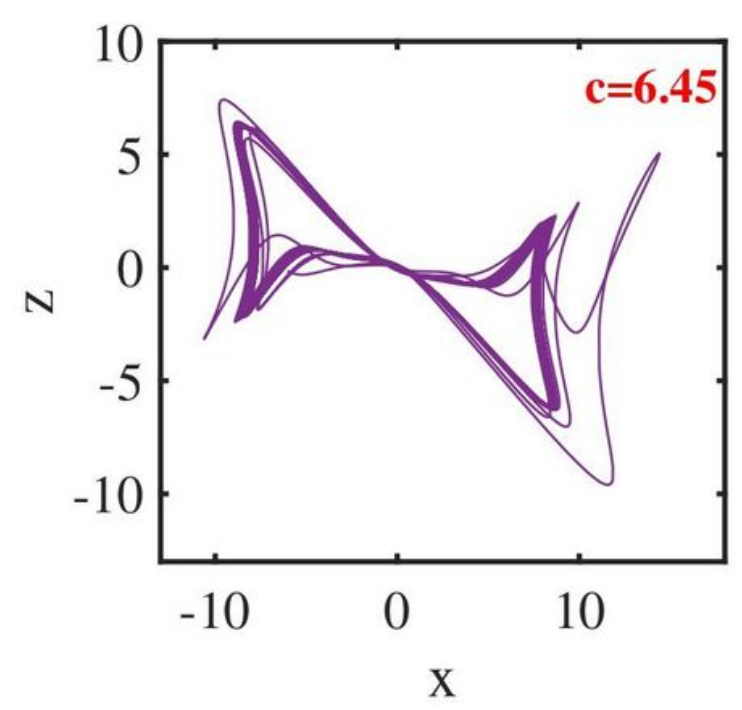

(d)

Figure 12

Please see the Manuscript PDF file for the complete figure caption 

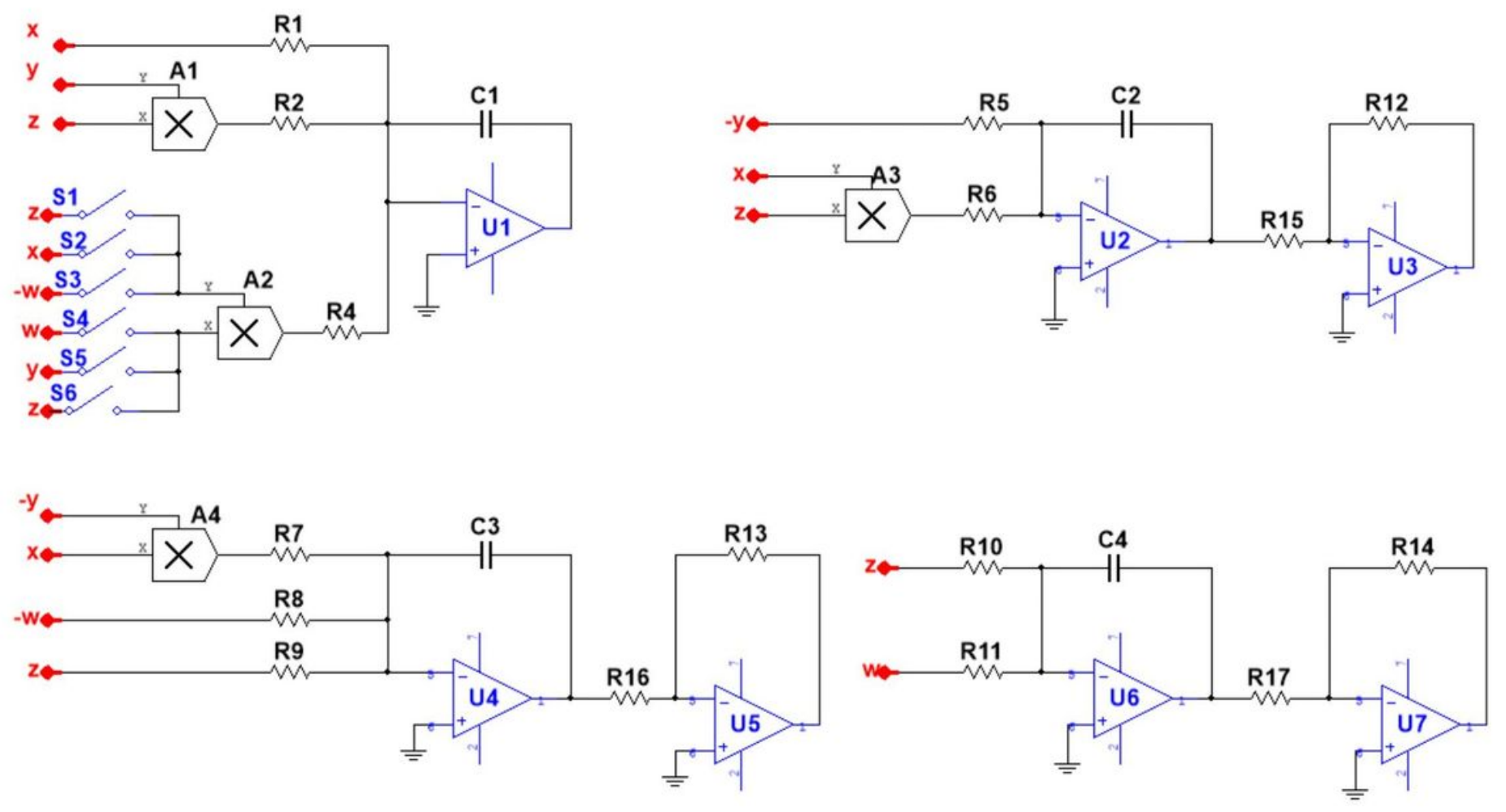

Figure 13

Please see the Manuscript PDF file for the complete figure caption

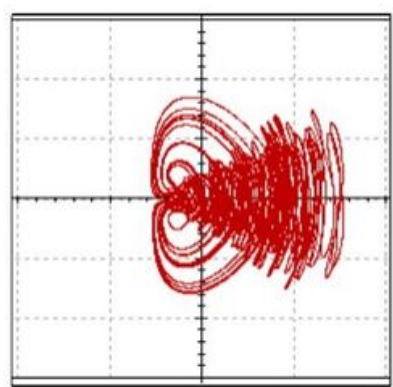

(a)

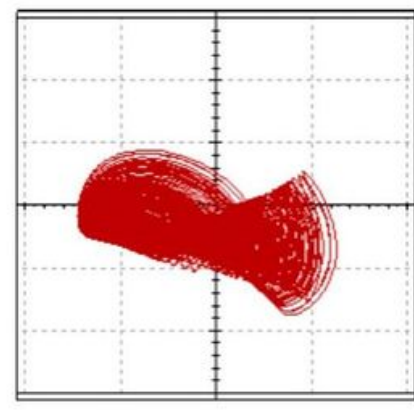

(b)

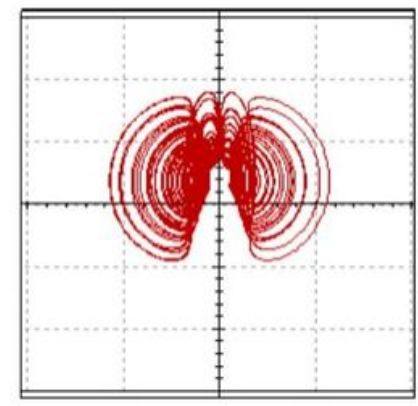

(c)

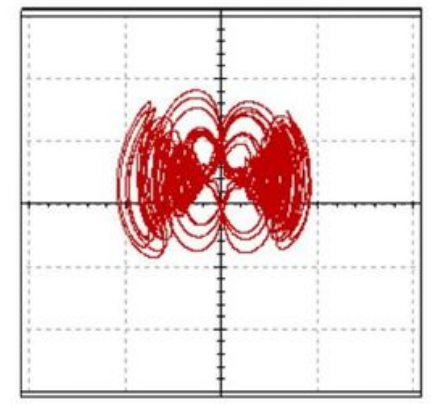

(d)

Figure 14

Please see the Manuscript PDF file for the complete figure caption 


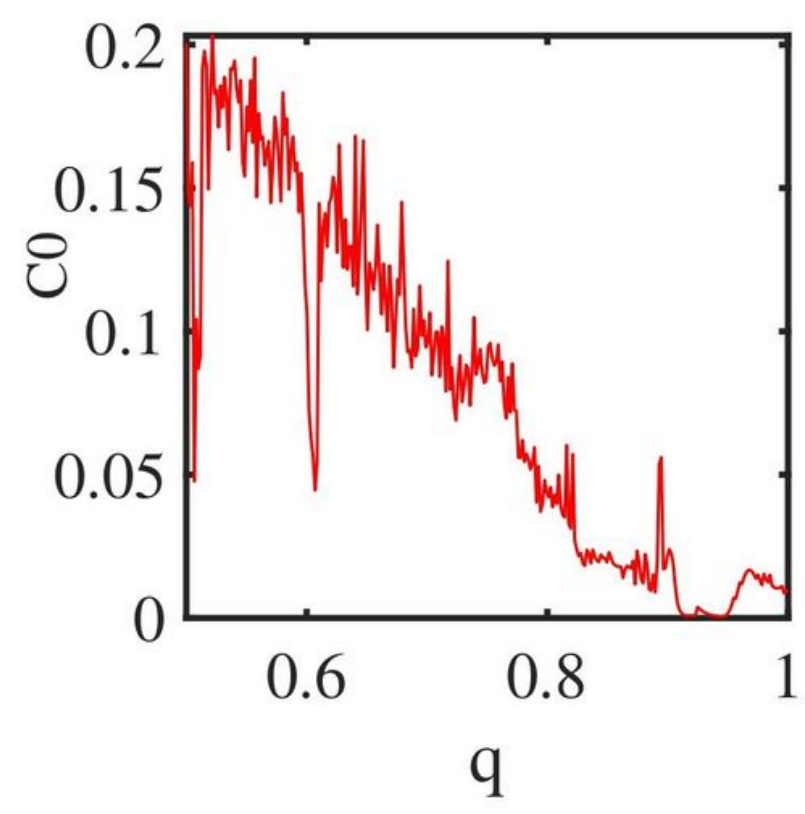

(a)

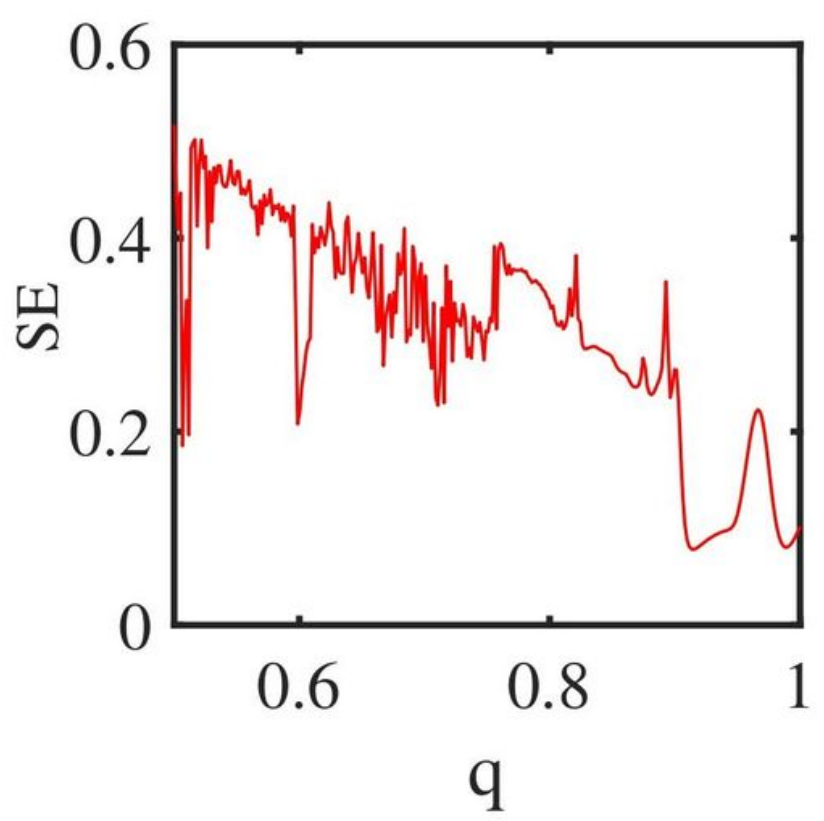

(b)

Figure 15

Please see the Manuscript PDF file for the complete figure caption

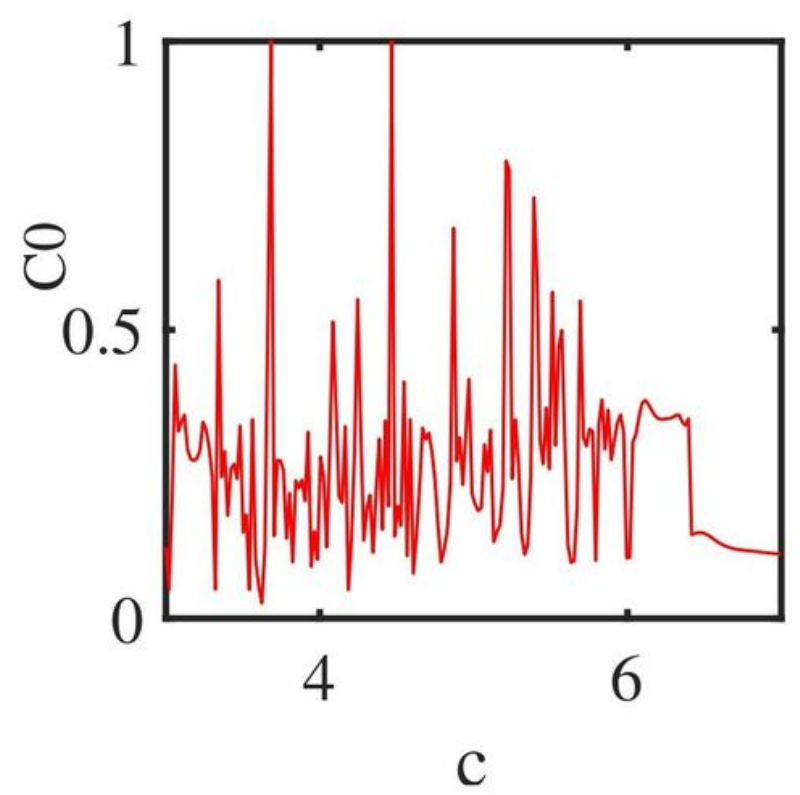

(a)

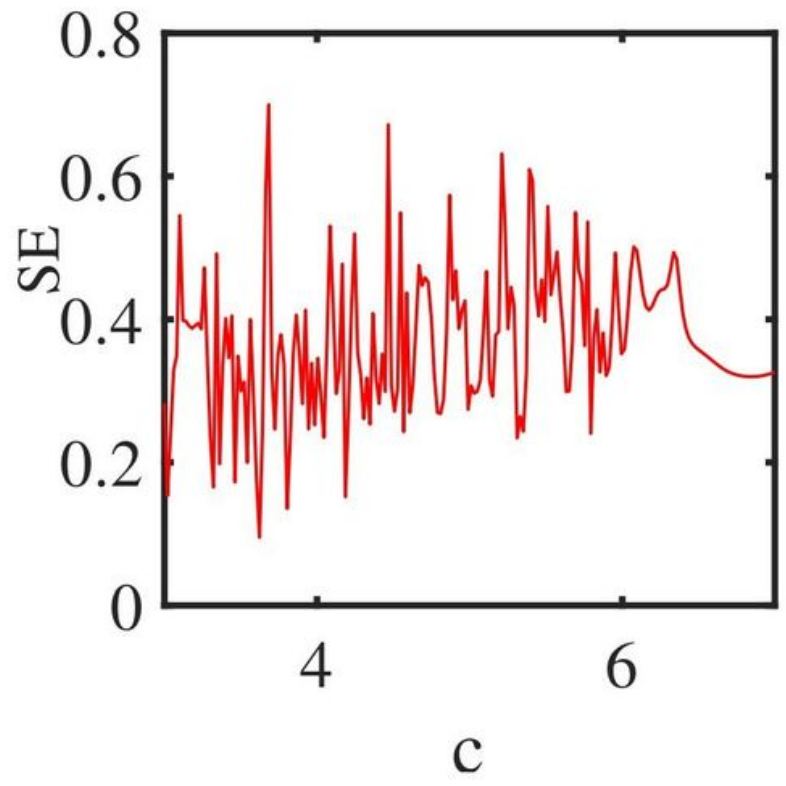

(b)

Figure 16

Please see the Manuscript PDF file for the complete figure caption 


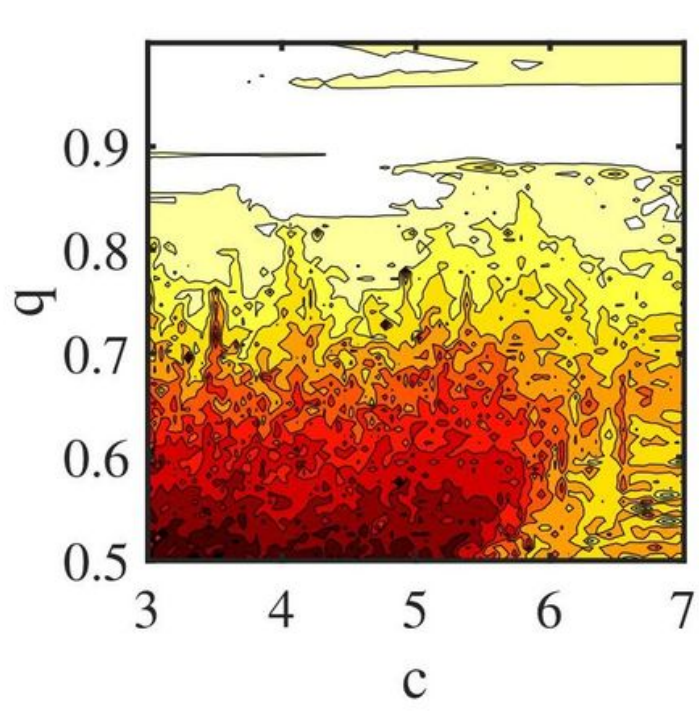

(a)

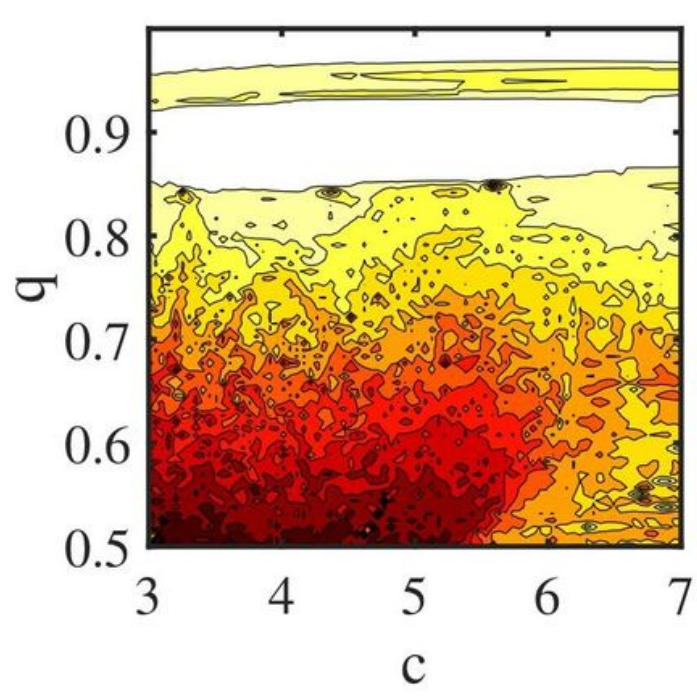

(c)

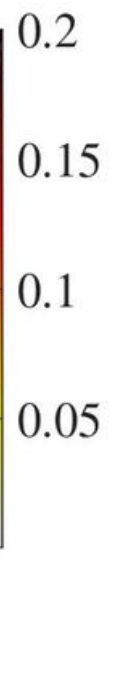

0.5

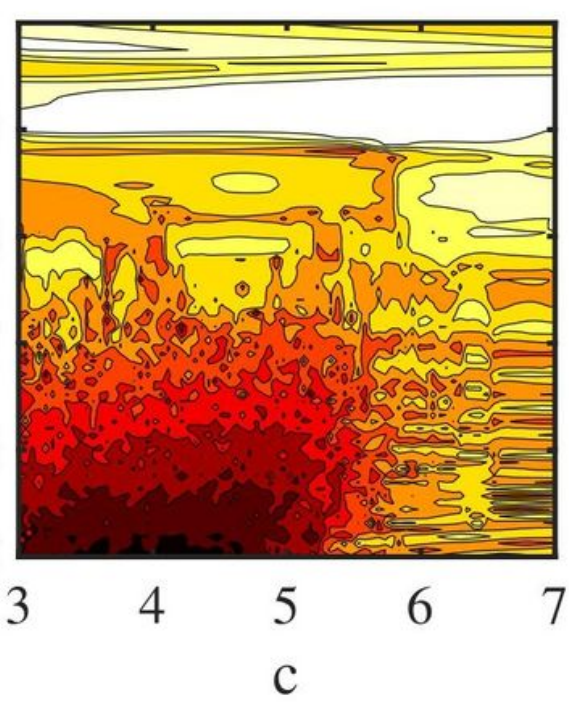

(b)
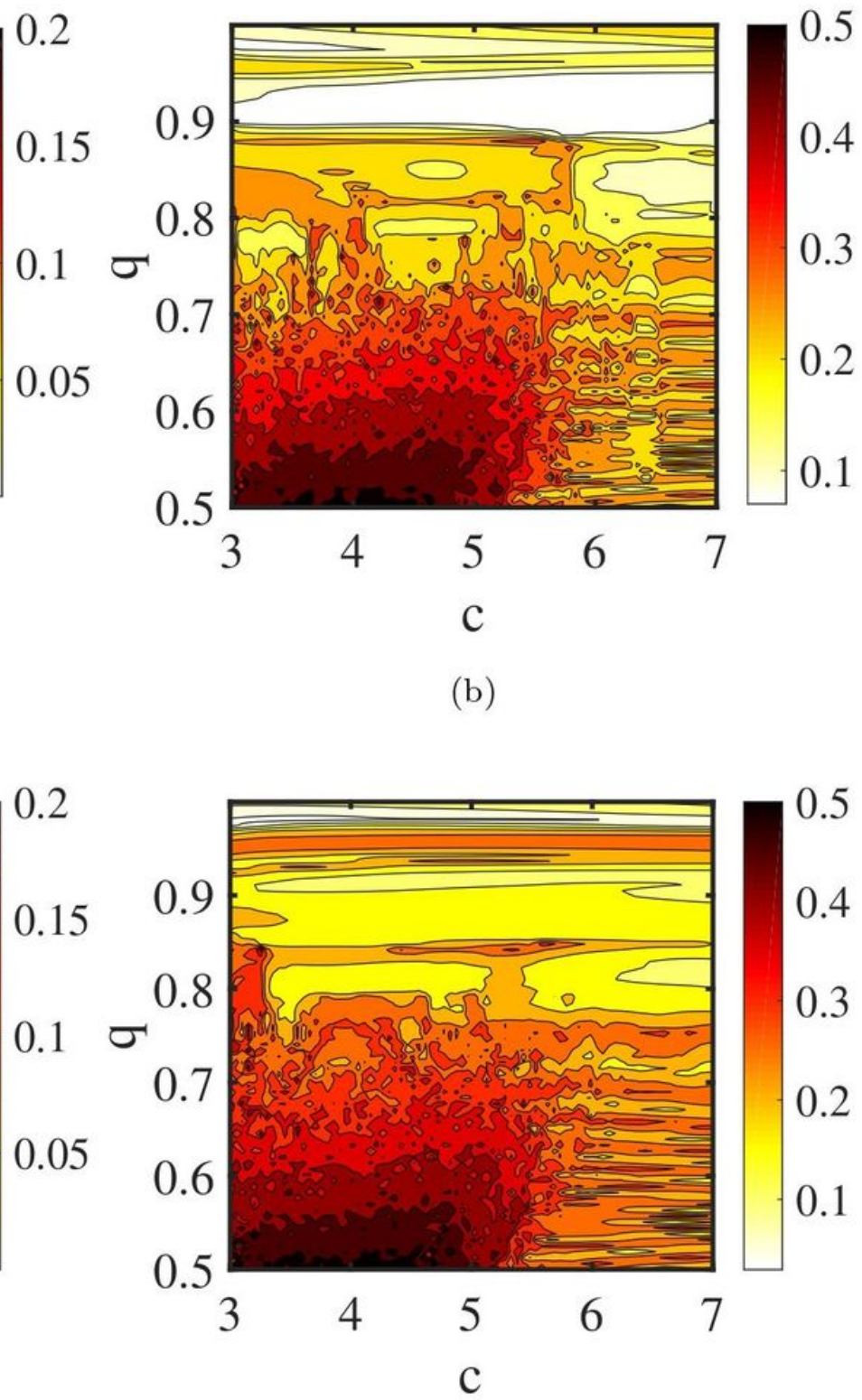

(d)

Figure 17

Please see the Manuscript PDF file for the complete figure caption 


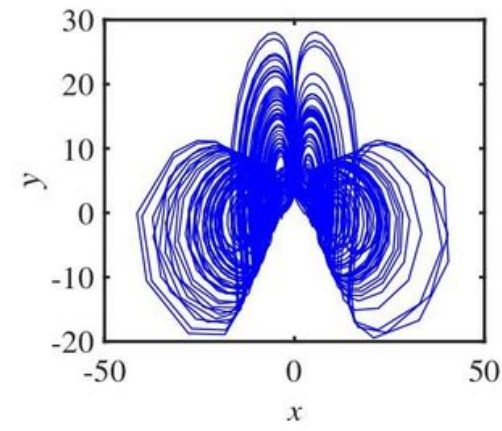

(a)

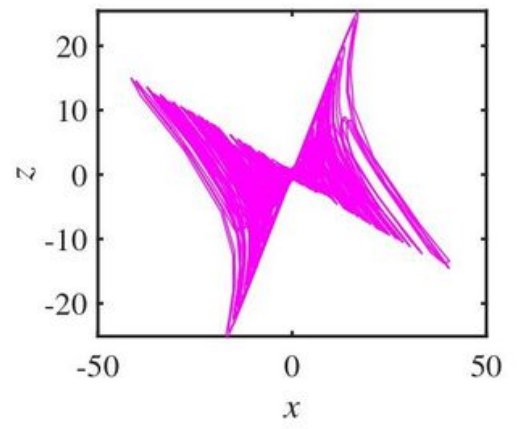

(b)

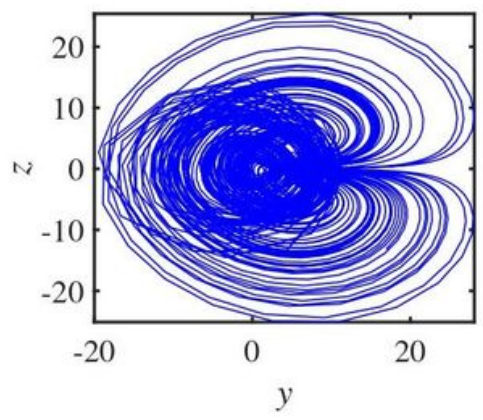

(c)

Figure 18

Please see the Manuscript PDF file for the complete figure caption

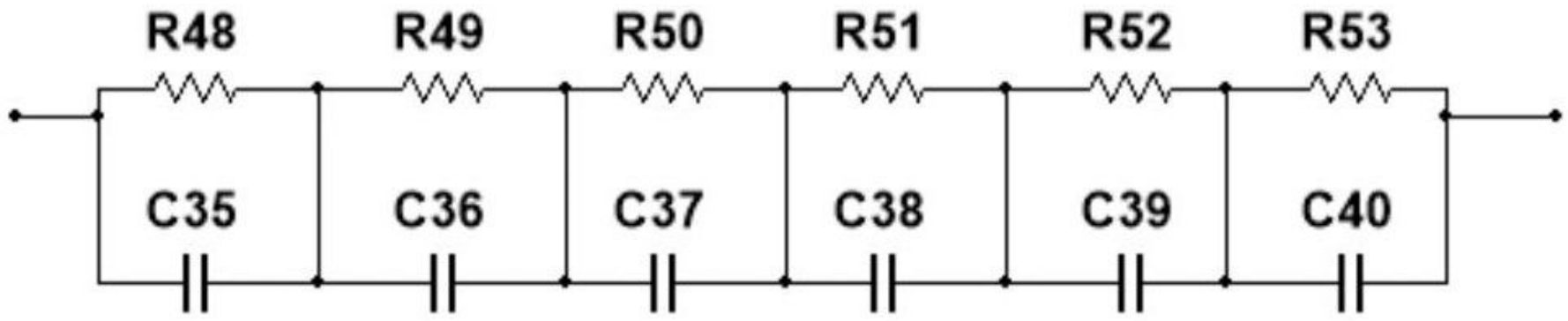

Figure 19

Please see the Manuscript PDF file for the complete figure caption

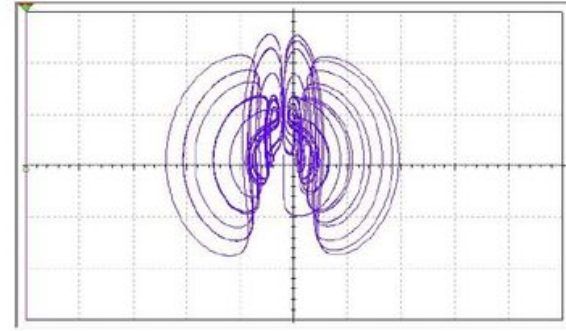

(a)

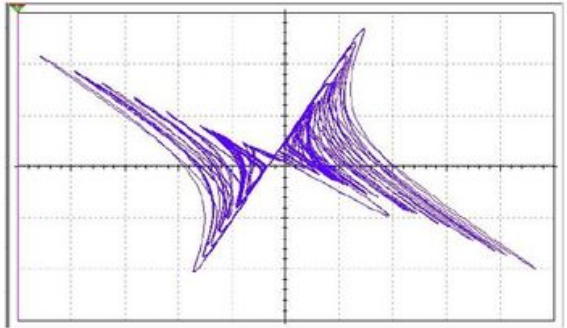

(b)

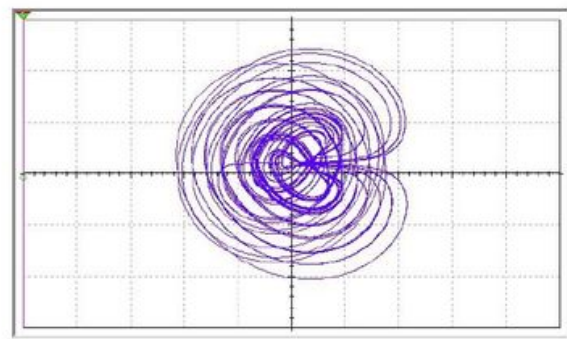

(c)

Figure 20

Please see the Manuscript PDF file for the complete figure caption 


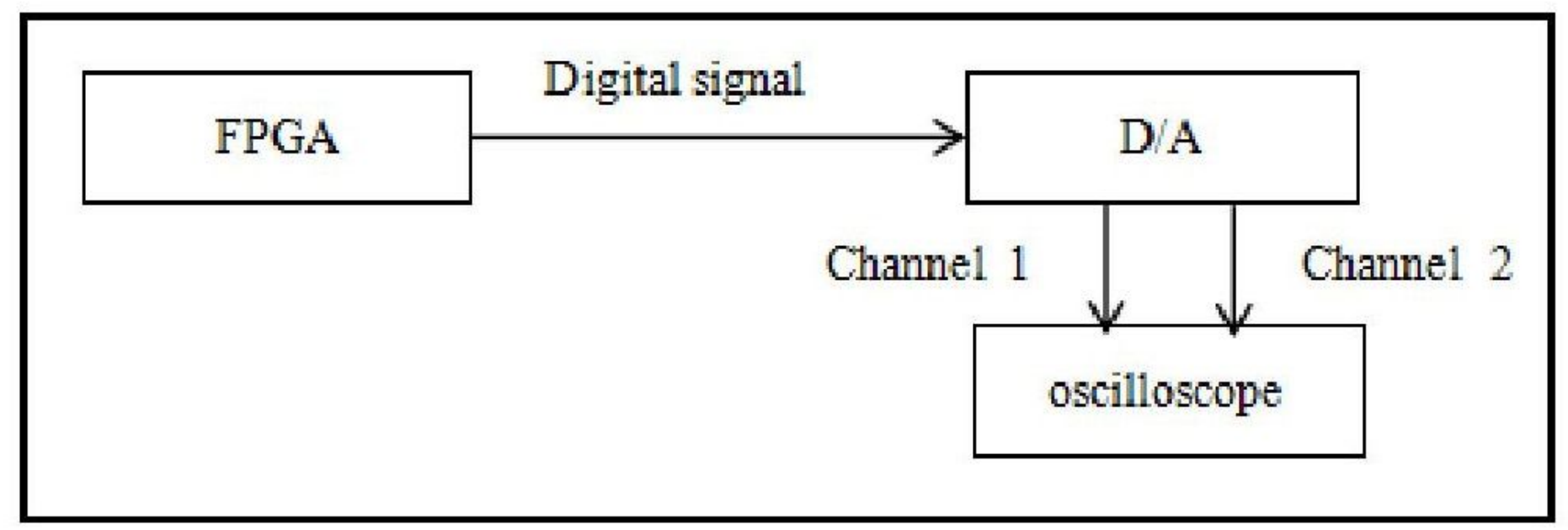

Figure 21

Please see the Manuscript PDF file for the complete figure caption
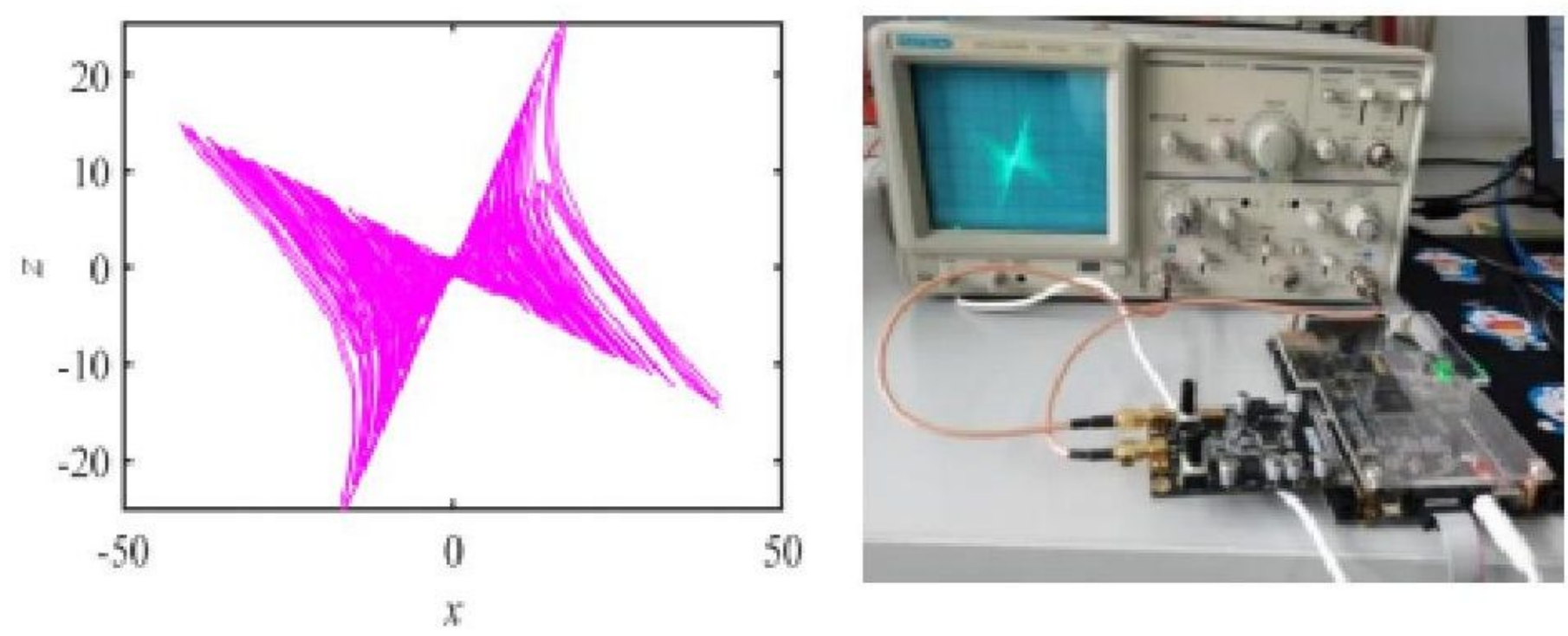

Figure 22

Please see the Manuscript PDF file for the complete figure caption 